\title{
L'ANALYSE DES DONNÉES APPLIQUÉE À LA TYPOLOGIE DES SITES GALLO-ROMAINS DANS LE BEAUCAIROIS (GARD) : MATÉRIEL DE PROSPECTION ET ENVIRONNEMENT PAYSAGER. ESSAI MÉTHODOLOGIQUE
}

\author{
par François FAVORY, Jean-Luc FICHES et Jean-Jacques GIRARDOT
}

Les travaux récents sur Ugernum $^{1}$ font mieux connaitre la topographie de la ville romaine de Beaucaire que les sources antiques présentaient comme un relais routier au Haut-Empire et une défense avancée d'Arles dans l'Antiquité tardive. Ils précisent les rapports d'Ugernum et de ses campagnes, définissent les limites et les réseaux du territoire, tentent d'approcher les structures agraires à travers l'occupation des sols et l'exploitation de l'espace rural.

Pour aborder la délicate question de l'habitat rural dans une zone de près de $150 \mathrm{~km}^{2}$ (fig. 1) qui correspond à peu près au canton actuel de Beaucaire (plus le territoire de Comps, moins ceux de Fourques et de Vallabrègues), nous disposions de 58 notices consacrées à des sites reconnus généralement par prospection ou à des découvertes fortuites. Seule en effet la villa des Tourelles-Pauvre Ménage a fait l'objet, dans cette région, de fouilles programmées en raison de la présence d'un atelier d'amphores ${ }^{2}$.

Dans cet ensemble de 58 notices, 43 comportaient des renseignements comparables qui permet-

1 J.-C. Bessac et al., Etudes sur Ugernum. Beaucaire el le Beaucairois à l'époque romaine, Associalion pour la Recherche Archéologique en Languedoc Oriental (A.R.A.L.O.), cahier n' 15, Caveirac, 1987. Les chapitres de la première partie, "Ville et campagnes" sont dus à J.-L. Fiches, Y. Gasco et A. Michelozzi. Cette étude doit beaucoup au travail de terrain de ces derniers.

2 Fouilles Fr. Widermann, Gallia, 41, 1983, p. 509; - F. Laubenheimer, La production des amphores en Gaule Narbonnaise, Paris, 1985, p. 131-135. taient d'y voir des habitats ${ }^{3}$. Certes, cet échantillonnage n'était pas le résultat de prospections systématiques et n'offrait pas d'homogénéité tant pour la quantité de matériel recueilli et conservé, que pour les terroirs et les sols, répartis entre plaine, coteau, garrigue et plateau des Costières. La carte archéologique était, en effet, le résultat de différentes opérations conduites à diverses époques : découvertes anciennes relocalisées, découvertes des années 60 à la suite des transformations culturales consécutives à l'aménagement du canal du Bas-Rhône, et découvertes récentes (1980-1985) à l'occasion de vérifications et d'enquètes orales.

Si elle représentait un handicap certain pour l'interprétation, cette hétérogénéité imposait une grande rigueur dans la caractérisation des sites. Les résultats publiés montrent l'intérêt de l'analyse : dans un secteur particulièrement bien prospecté, on a pu cerner, par exemple, l'étendue de domaines du Haut-Empire, pour certains d'origine indigène, qui avaient été exceptés d'une première cadastration (Orange A) avant d'être intégrés, vraisemblablement sous Auguste, dans une seconde centuriation (Nîmes B). Mais plus que sur les résultats, nous voudrions ici insister sur la démarche méthodologique, les descripteurs des sites et la définition des classes et de leurs subdivisions, qui ont permis d'aboutir à de telles conclusions.

3 Les sites ont conservé ici la numérotation des notices publiées dans Études sur Ugernum ... op. cil. La sélection de 43 notices explique les manques perceptibles dans les tableaux et les graphiques. On trouvera l'inventaire des sites retenus en appendice. 


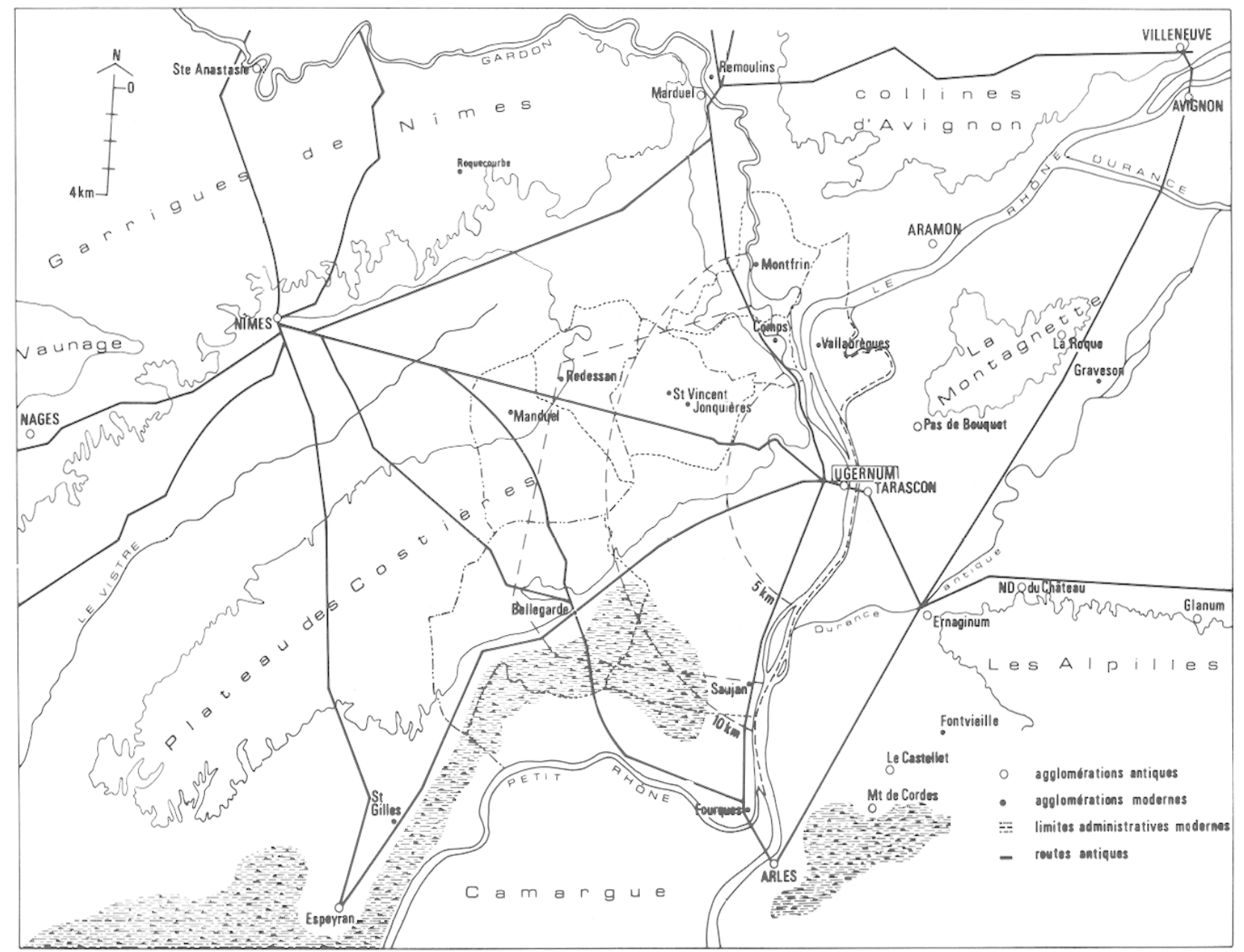

Fig. 1 - Carte de situation.

\section{LES CHOIX LIMINAIRES}

\section{Choix de critères de classification}

En abordant le Beaucairois, nous pouvions nous référer aux typologies de sites d'époque romaine, élaborées surtout à partir des programmes de prospections conduits par les anglais en Italie ${ }^{4}$. Le contexte était pourtant radicalement différent: des vestiges de surface beaucoup plus détruits et l'absence quasi totale de fouilles de référence interdisaient d'interpréter d'emblée un site en fonction du matériel livré et de sa superficie.

4 On trouvera la bibliographie et l'historiographie de cette question dans les contributions de T. W. Potter, M.-G. Celuzza et E. Fentress dans l'ouvrage de A. Ferdière et E. Zanora-Rio, la prospection archéologique: paysage el peuplement, Documents d'Archéologie Française, 3, Paris, 1986, p. $111-120$ et 139-144.
Nous avons donc tenté de définir des descripteurs en fonction des données propres du terrain, à partir de la qualité du matériel recueilli, de la superficie mais aussi de l'implantation du site, le choix étant commandé par le souci d'utiliser au maximum une documentation maigre et peu différencićc.

Les indices révélateurs d'un site archéologique donnent des précisions sur sa structure et sur sa chronologie. Parmi ces indices, certains rendent compte des matériaux de construction utilisés, d'autres du mode de vie, d'autres éventuellement d'activités spécifiques (agricoles ou artisanales). Les indicateurs chronologiques permettent d'appréhender, de façon plus ou moins précise, la durée d'occupation et la date d'implantation. On a donc distingué cinq critères de caractérisation à partir de l'analyse du matériel: matériaux, vie, activité, occupation et date.

Avec la surface d'épandage des vestiges (superfi- 
cie), on a retenu cinq critères en rapport avec la situation du site : la nature du sol (sol), le terroir, l'orientation de la pente (pente), la distance par rapport à la voirie principale et au Rhône et l'orientation du parcellaire autour du site. On n'd pas retenu de critère sur les ressources en eau étant donné les facilités de l'approvisionnement dans toute la région.

\section{DÉFINITION DES DESCRIPTEURS ET DE LEURS MODALITÉS}

L'information relative à chacune des 43 notices retenues pour l'analyse a donc été exploitée en fonction de ces onze descripteurs, dont l'éclatement booléen selon leurs diverses modalités initiales (catégories ou classes) a généré au total 41 caractères. On trouvera ci-après la définition de chaque descripteur et de ses modalités ainsi que leurs codes. La figure 2 les présente sous la forme d'un graphique de pourcentages.

1. Superficie : On avait initialement distingué six classes de surface d'épandage du matériel. Les deux extrêmes n'apparaissent pas sur le tableau en raison de leur faible représentation : deux sites de moins de 0,1 ha et trois sites de plus de 5 ha. Cette nouvelle répartition estompe le petit nombre des gisements de faible étendue. Ailleurs, on a généralement observé que le nombre de sites était inversement proportionnel à leur surface; ainsi, en Navarre, $35 \%$ des sites ont moins de 0,5 ha $; 20 \%$ de 0,5 à 1 ha; $15 \%$ de 1 à 1,5 ha et $5 \%$ plus de 1,5 ha $^{5}$. Dans l'ager cosanus, la répartition est la suivante : plus de 50 sites de moins de 0,25 ha; douze entre 0,25 et 0,5 ha; seize entre 0,5 et 1 ha; quinze entre 1 et 2,5 ha; treize de plus de 2,5 ha $^{6}$.

Dans le Beaucairois, le faible nombre des sites de moins de 0,5 ha et surtout de moins de 0,1 ha par rapport à ceux de la classe immédiatement supérieure, s'explique aisément par le caractère non systématique de la prospection; on notera que la majeure partie des sites de faible superficie ont été trouvés récemment, et l'on peut penser que les sites identifiables de ce type sont en fait beaucoup plus nombreux. On peut admettre également, au regard de l'histoire de la prospection de cette région, que la proportion connue des sites de grande superficie est plus

5 J.-L. ONa Gonzal.Fs, El poblementio rural de época romana en una zona de la ribera de Navarra, Arqueología espacial, 5, Teruel, 1984, p. 89.

6 M.-G. Gricuzza et E. Fentress, L'occupation du sol dans l'ager cosanus et la vallée de l'Albegna (Italie), dans $L a$ prospection archéologique ..., op. cil., p, 117-118. importante. On retiendra donc comme plus significatif le nombre particulièrement élevé de sites dont la surface est supérieure à 2 ha.

2. Matériaux: Une large majorité de sites du Beaucairois n'a livré, comme matériaux de construction, que des tuiles et des moellons (Mat. 1). On n'en connaît aucun sur lesquels de tels indices soient absents, et qui pourraient donc témoigner de constructions uniquement en terre et en bois.

Beaucoup moins nombreux sont les sites qui ont livré d'autres matériaux. On a distingué d'une part ceux qui témoignaient de l'usage du mortier de chaux (enduits peints, bétons de tuileaux) ou de la terre cuite (briques, tubuli, pilettes d'hypocaustes...) (Mat. 2), et d'autre part ceux qui fournissaient en outre des éléments architectoniques, des cubes de mosaïques et du marbre (Mat. 3).

3. Mode de vie: Pour évaluer la "richesse» des gisements, on a distingué ceux qui ont fourni de la vaisselle fine (céramiques et verrerie) (Vie 2 ), ceux sur lesquels on a recueilli des monnaies et du petit mobilier (Vie 3), et ceux enfin qui se distinguent par la présence d'éléments peu courants : ici, sculpture ou inscription votive; ailleurs, trésor monétaire, objet d'art... (Vie 4): on a classé dans le groupe Vie 1 les sites n'ayant livré que des amphores et des dolia.

Comme pour le descripteur précédent, la répartition est très inégale et la grande majorité se situe dans les groupes 2 et 3 qui correspondent à la situation la plus banale.

4. Activité : Les activités qui peuvent s'inscrire dans le cadre domestique, ont été subdivisées en deux catégories en fonction du matériel : on a considéré d'une part que la présence d'amphores et de dolia marquait une activité de stockage (Act. 1); c'est le cas le plus fréquent. On a pris en compte d'autre part, la présence de petit outillage ou d'instrument mobiliers à caractère artisanal (truelle, peson ...) ou agricole (meule à bras) (Act. 2).

Les sites qui fournissent des indices d'installations spécialisées (fours, pressoirs, celliers ...) (Act. 3) restent exceptionnels.

5. Durée d'occupation: En raison de l'inégalité et surtout de la faiblesse des échantillons de matériel, on a été contraint à une approche grossière. Il n'a pas été possible d'isoler des sites à occupation unique et ponctuelle (pas plus d'un demi-siècle) et de percevoir des hiatus dans les sites occupés plus durablement. On a distingué surtout les sites qui ont livré des documents du Haut et du Bas-Empire (Occ. 2) et ceux qui en ont livré seulement pour le 
1 Superficie:

$\mathrm{S}<.5 \quad$ inférieure à un demi-hectare

$\mathrm{S}<1 \quad$ comprise entre 0,5 et 1 ha

S1-2 comprise entre 1 et 2 ha

$\mathrm{S}>2$ supérieure à $2 \mathrm{ha}$

2 Matériaux:

Mat1 tuiles et moellons

Mat2 mortier de chaux, terre cuite

Mat3 mosaïque, marbre, élément architectonique

3 Mode de vie:

Vie1 amphore, dolium

Vie2 vaisselle fine

Vie3 petit mobilier, monnaies

Vie4 sculpture, inscription, trésor monétaire ...

4 Activité:

Act1 stockage domestique

Act2 instruments et outils manuels

Act3 installations spécialisées

5 Durée d'occupation:

Occ1 Haut-Empire

Occ2 Haut et Bas-Empire

6 Date d'implantation:

HEmp Haut-Empire

Aug Epoque augustéenne

Iav Ier s. av. J.-C.

7 Sol:

Sol1 sablo-limoneux

Sol2 complexe de piedmont

Sol3 pauvre sur roche mère

8 Terroir:

Ter1 plaine

Ter2 côteau

Ter3 plateau des Costières

Ter4 garrigue des collines de Beaucaire

9 Pente:

Pnul pente nulle

PE orientée à l'Est

PSSE orientée au Sud-Sud-Est

PW orientée à l'Ouest

PN orientée au Nord

10 Distance à la voirie:

$<50$ inférieure à $50 \mathrm{~m}$

$<150 \quad$ comprise entre 50 et $150 \mathrm{~m}$

$<300$ comprise entre 150 et $300 \mathrm{~m}$

$<500$ comprise entre 300 et $500 \mathrm{~m}$

$<1 \mathrm{~km} \quad$ comprise entre $500 \mathrm{~m}$ et $1 \mathrm{~km}$

$>1 \mathrm{~km}$ supérieure à $1 \mathrm{~km}$

11 Orientation du parcellaire:

$\begin{array}{ll}\text { C2.5 } & \text { N 2 }{ }^{\circ} 5^{\prime} \mathrm{E} \\ \text { C. } 10 & \mathrm{~N} 10^{\circ} 30^{\prime} \mathrm{W} \\ \text { C. } 20 & \mathrm{~N} 20^{\circ} \mathrm{W} \\ \text { C. } 32 & \mathrm{~N} 32^{\circ} \mathrm{W}\end{array}$

16 ind. $\quad 37 \%$

13 ind. $30 \%$

5 ind. $11 \%$

9 ind. $20 \%$

* $* * * * * * * * * *$

$* * * *$

$* * * * * * * *$

27 ind.

6 ind. $13 \%$

10 ind. $23 \%$

*************************

$* * * *$

$* * * * * * * * *$

4 ind. $\quad 9 \%$

25 ind. $\quad 58 \%$

11 ind. $\quad 25 \%$

3 ind. $6 \%$

$* * *$

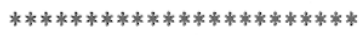

$* * * * * * * * * *$

$* *$

30 ind. $\quad 69 \%$

9 ind. 20\%

3 ind. $6 \%$

$* * * * * * * *$

$* *$

27 ind $\quad 62 \%$

15 ind. $\quad 34 \%$

$* * * * * * * * * * * * *$

29 ind. $\quad 67 \%$

5 ind. $11 \%$

8 ind. $18 \%$

$* * * * * * *$

11 ind. $\quad 25 \%$

25 ind. $\quad 58 \%$

7 ind. $16 \%$

$* * * * * * * * * *$

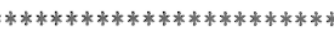

$* * * * * *$

16 ind. $\quad 37 \%$

14 ind. $\quad 32 \%$

10 ind. $23 \%$

$* * * * * * * * * * * * * *$

3 ind. $6 \%$

***k*k****

$* *$

$\begin{array}{rrl}9 \text { ind. } & 20 \% & * * * * * * * * \\ 20 \text { ind. } & 46 \% & * * * * * * * * * * * * * * * * * * \\ 8 \text { ind. } & 18 \% & * * * * * * * \\ 2 \text { ind. } & 4 \% & * \\ 4 \text { ind. } & 9 \% & * * *\end{array}$

$\begin{array}{rrl}9 \text { ind. } & 20 \% & * * * * * * * * \\ 10 \text { ind. } & 23 \% & * * * * * * * * * \\ 7 \text { ind. } & 16 \% & * * * * * * \\ 3 \text { ind. } & 6 \% & * * \\ 4 \text { ind. } & 9 \% & * * * \\ 10 \text { ind. } & 23 \% & * * * * * * * * *\end{array}$

11 ind.

7 ind. $16 \%$ ******

5 ind. $11 \% \quad * * * *$

4 ind. $9 \% \quad * * *$

Fig. 2 - Édition du comptage des caractères (pourcentages arrondis). 
Haut-Empire (Occ. 1). Seul le site 19, qui n'apparaît pas sur le tableau à ce niveau, a eu une occupation limitée à l'Antiquité tardive. Ce gisement n'est vraisemblablement pas aussi exceptionnel qu'il y paraît; lès ramassages sélectifs - surtout les plus anciens - ont certainement privilégié la reconnaissance des sites du Haut-Empire sur lesquelles la céramique sigillée attirait l'attention.

Ce critère de la continuité dans l'implantation de l'habitat n'est certainement pas celui de la permanence de ses structures et de ses fonctions qui ont pu varier durant l'Antiquité. Les recherches sur l'habitat rural du Var où des fouilles importantes ont pu être réalisées récemment, témoignent de ces changements dans le Midi de la Gaule ${ }^{7}$; elles rendent compte aussi d'un phénomène de concentration de l'habitat au III's., maintes fois souligné ailleurs et que l'on retrouve autour de Beaucaire. $\mathrm{Si}$, dans ce cas, la proportion de sites qui livrent des vestiges de l'Antiquité tardive est importante $(40 \%)$, on n'oubliera pas de relativiser ce chiffre en considérant que cet échantillon comporte un petit nombre de sites de faible superficie dont la plupart sont généralement abandonnés au Bas-Empire.

6. Date d'implantation: Dans ce cas encore la faiblesse de l'échantillon a commandé une division grossière. On a également été prudent en raison des incertitudes qui pèsent sur la datation de plusieurs catégories de céramiques qui constituent les principaux fossiles-directeurs. Le petit nombre de sites enfin a interdit de créer des classes trop faiblement représentées. Le site 19 mis à part en raison de son occupation limitée à la seconde moitié du IV $\mathrm{V}^{\mathrm{e}}$., on a distingué ceux qui ont livré de la céramique campanienne ( $\mathrm{I}^{\mathrm{er}} \mathrm{s}$. av. J.-C., $\mathrm{n}^{\text {s }} 7,9,14,16,23,26$, 34,44 ) et de la céramique arétine (époque augustéenne, $\left.\mathrm{n}^{\text {os }} 10,12,20,43\right)$, du lot le plus important qui a été rattaché au Haut-Empire sans plus de précision.

7. Sol: A partir des indications de la carte géologique au $1: 50000^{\mathrm{e}}$, on a distingué les sols sablolimoneux (Sol 1 : alluvions des vallées du Rhône et du Gardon, et limons lossiques ou grès des Costières), les complexes des formations de piedmont qui mêlent sables, limons, cailloux et galets (Sol 2) et les sols pauvres sur roche mère (Sol 3 : grès des Costières et calcaire des garrigues).

7 J.-P. Brun et al., L'habitat rural dans le Var à l'époque romaine : données archéologiques récentes, Provence Historique, 141, 1985, p. 233-251.
8. Terroir : On a associé ce critère au précédent car la distinction entre plaine (Ter. 1), coteaux (Ter. 2), plateau des Costières (Ter. 3) et collines de Beaucaire (Ter. 4) ne recouvre pas exactement les catégories des sols. $\Lambda$ insi, les sols 1 se partagent entre Ter. 1 et 3 et les sols 3 entre Ter. 3 et 4 .

9. Pente: La direction de la pente du terrain a été codée en utilisant la numérotation d'un cadran de montre dont le 12 correspondait au Nord. On a ensuite procédé aux regroupements suivants : $\mathrm{PN}=1,11,12 ; \mathrm{PW}=8,9,10 ; \mathrm{PE}=2,3,4 ;$ $\mathrm{PS}=5,6,7$ (les orientations 6 et 7 étant absentes, on a désigné ce groupe par PSSE).

Une large majorité de sites est exposée à l'e. et au s.-e. conformément aux grandes lignes du relief de la région. Les deux qui sont exposés à l'o., sont situés à l'o. de la garrigue, près de la dépression de Jonquières. Ceux, également rares, qui sont mentionnés sur la pente n., sont des sites de plaine ou de plateau où la pente est donc très faible.

10. Distance à la voirie: L'évaluation sur la carte de la distance des sites par rapport au Rhône et à la voirie principale (fig. 1 : voie domitienne et routes de piedmont de Costières) a fait distinguer six classes limitées par les distances suivantes : $50 \mathrm{~m}$, $150 \mathrm{~m}, 300 \mathrm{~m}, 500 \mathrm{~m}$ et $1 \mathrm{~km}$. La répartition des sites entre ces classes est relativement équilibrée. Cependant, $57 \%$ se trouvent à moins de $300 \mathrm{~m}$ d'une voie et $65 \%$ à moins de $500 \mathrm{~m}$.

11. Orientation du parcellaire: On a enfin pris en considération l'orientation dominante du parcellaire dans l'environnement immédiat de chaque site. On a retenu quatre orientations bien représentées, qui concernent 22 gisements $(51 \%$ : quatre sites sont concernés par deux orientations). Le parcellaire le plus structurant ( $2^{\circ} 5^{\prime} \mathrm{E}$ ) de l'habitat $(25 \%)$ s'inscrit dans le système du cadastre A d'Orange (sites 4, 8,11 à $13,21,22,27,37,41,44$ ) qui concernait le territoire de plusieurs centres antiques dont Ugernum $^{8}$. Viennent ensuite les traces correspondant au cadastre Nîmes $\mathrm{B}\left(\mathrm{N} 10^{\circ} 30^{\prime} \mathrm{W}\right)$ avec les sites 14,22 , $26,35,41$ et 43. Les autres orientations sont moins fréquentes et se rapportent à des sites groupés sur le coteau et aux abords; elles sont vraisemblablement commandées par le relief : $\mathrm{N} 20^{\circ} \mathrm{W}$ pour les sites 9 , $10,30,57$ et $58 ; \mathrm{N} 32^{\circ} \mathrm{W}$ pour les sites $18,28,30$ et 37.

8 G. Chouquer, Localisation et extension des cadastres affichés à Orange, dans Cadastres el espace rural, Paris, 1983, p. 288. Pour le cadastre Nimes B, voir, dans le même ouvrage, la contribution de J.-L. Fiches et J. Soyer. 


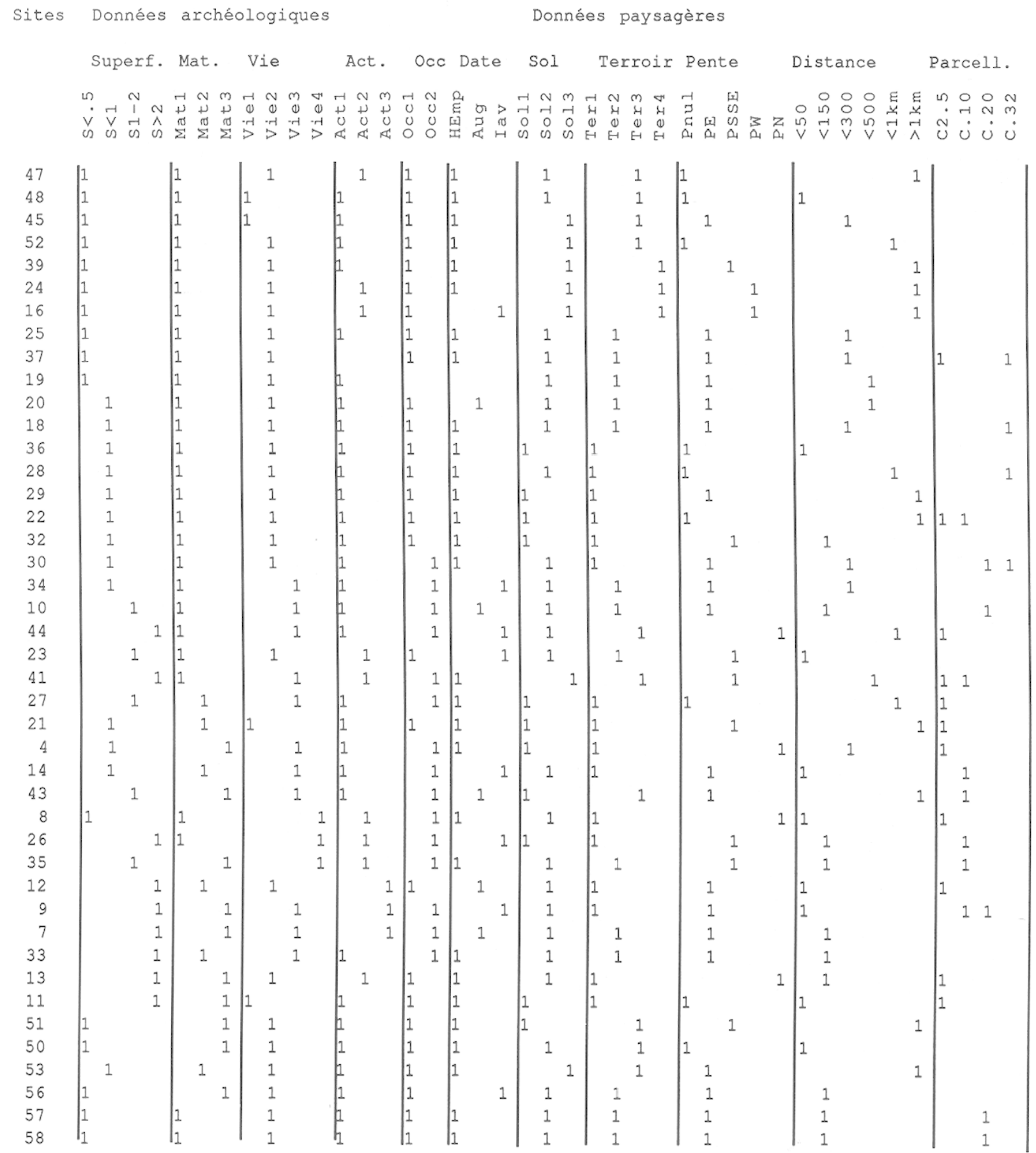

Fig. 3 - Tableau des données éclatées en caractères booléens suivant les matrices Bertin.

Premier fasai de classification : DIAGONALISATION DES DONNÉES

Le premier essai de classification a été réalisé à la main selon une diagonalisation des données inspirée des matrices Bertin ${ }^{9}$. Ainsi s'explique l'ordre

9 J. Bertin, Sémiologie graphique, Paris, 1967 ; Représentation graphique, Encyclopaedia Universalis, s.v. Graphique. des sites qui a été enregistré pour les analyses suivantes (fig. 3). La comparaison de ce résultat intermédiaire avec l'interprétation finale fait d'ailleurs apparaître des différences, dues notamment au poids particulier accordé alors de façon trop systématique à la superficie des sites.

A ce stade du travail, nous ressentions les limites d'une approche de ce type et nous éprouvions le besoin de tester la validité et le poids respectif des 
descripteurs retenus. Voilà pourquoi nous avons eu recours à l'analyse factorielle des correspondances et à la classification ascendante hiérarchique qui font l'objet des principaux développements de cet article.

Il n'est pas question ici de comparer le produit, d'une démarche non aboutie, faute de maîtrise suffisante de l'outil utilisé, et les résultats d'une analyse des données conduite à son terme : on se bornera à remarquer que nombre d'archéologues tentent d'utiliser de manière empirique cette technique, et, en tout état de cause, ce tableau illustre le point atteint dans la manipulation des informations, sans qu'il ait été possible d'aller au-delà.

\section{L'ANALYSE FACTORIELLE DES CORRESPONDANCES (AFC)}

Les deux graphes (fig. 4 et 5) donnent une représentation des résultats de l'analyse factorielle des correspondances opérée ici avec sélection de trois facteurs ${ }^{\mathbf{1 0}}$.

Chacun des graphes représente des relations qu'entretiennent entre eux les 43 sites en fonction de leurs rapports avec les caractères qu'ils partagent ou non. Représentation déformée dans la mesure où sites et caractères sont projetés sur le plan du graphe, en fonction de leur dispersion dans l'espace factoriel à trois dimensions. A l'origine, le tableau permet la représentation graphique des sites dans un espace à 41 dimensions et celle des caractères dans un espace à 43 dimensions. L'analyse factorielle des correspondances nous permet d'appréhender la répartition des deux ensembles de points au sein d'un nuage représentable dans un espace réduit, mais perceptible. Ainsi tous les points sont représentés dans le plan, même si, dans le volume du nuage, ils sont éloignés de ce plan : la multiplicité des graphes et les aides à l'interprétation que le logiciel ANACONDA ${ }^{11}$ permet d'éditer, offrent le moyen de corriger ces effets de parallaxe.

10 J.-P. BrnzícRI et al., L'analyse des données, I. La taxinomie; II. L'analyse des correspondances, Dunod, Paris, 1980 ; - J.-P. FÉnELION, Qu'est-ce que l'Analyse des données?, Lefonen, Paris, 1981; - sur les travaux du Laboratoire MIS de Besançon: J.-Ph. Massonié, Le MIS "Mathématique, informatique, statistique" : un laboratoire au service de la recherche littéraire, Compuler and the Humanities, 20, 1986, 3, p. 193-195.

11 Créé par J.-J. Girardot : Anaconda, Cahiers du Séminaire Universitaire de Recherches Floues du Laboratoire MIS, 1, 1982, Université de Franche-Comté, Besançon. Une partie des programmes utilisés pour conduire cette analyse a fonctionné en version expérimentale.
Après prise en compte de ces limites à l'apparente cohérence spatiale des relations représentées dans chacun de ces graphes, et après examen des divers niveaux de structuration des regroupements et des différenciations d'ensembles de points, il est possible de dégager la signification de chacun des axes (1,2 et 3 ), concrétisant les facteurs qui ont organisé la distribution des sites et des caractères.

\section{L'AXE 1}

Dans les graphes présentés ici, l'axe 1 est horizontal. L'axe 1 est le facteur utile dont l'inertie est la plus forte, c'est-à-dire l'axe qui est déterminé par l'extension maximale du nuage : les points se dispersent plus le long du premier axe que des suivants, et ainsi de suite. Il exprime donc l'information la plus décisive pour comprendre la structure du nuage, traduite par l'organisation des points sur les graphes.

Si l'on considère les deux ensembles regroupés aux deux pôles de l'axe 1 , on note, à gauche, le regroupement des sites de superficie médiocre, de faible épandage des vestiges, livrant un mobilier banal, d'occupation restreinte pour une installation généralement datée du Haut-Empire. De ce groupe, s'extériorise un sous-groupe qui réunit, de manière quasi caricaturale, les caractères les plus représentatifs de cet ensemble de sites modestes : ce sont les sites de garrigue, installés sur des sols grossiers, loin des voies de communication.

A l'opposé, en haut à droite de l'axe, on recense les sites de superficie supérieure à 2 ha $(\mathrm{S}>2)$ et à 1 ha (S 1-2). Ces mêmes sites livrent le mobilier le plus riche, le plus varié et le plus significatif en ce qui concerne les activités économiques. On y trouve des sites implantés dès la République tardive et l'époque augustéenne et occupés pendant une longue durée. Ils sont localisés non loin des voies majeures, vecteurs des échanges, plutôt en plaine et sur la cuesta.

L'axe 1 a donc tendance à opposer les sites à matériel de classe 1 aux autres: mais on voit d'emblée les effets induits par un tel système antinomique. Qui dit matériel banal dit niveau de vie modeste, information sur l'activité limitée aux témoignages de stockage, etc.

\section{L'AXE 2 (fig. 4)}

Il tend à différencier les sites, déjà organisés par l'axe 1 selon le critère de la nature du mobilier, en fonction de leurs activités spécialisées. On trouve en effet, en haut du graphe, les sites à activité de niveau 


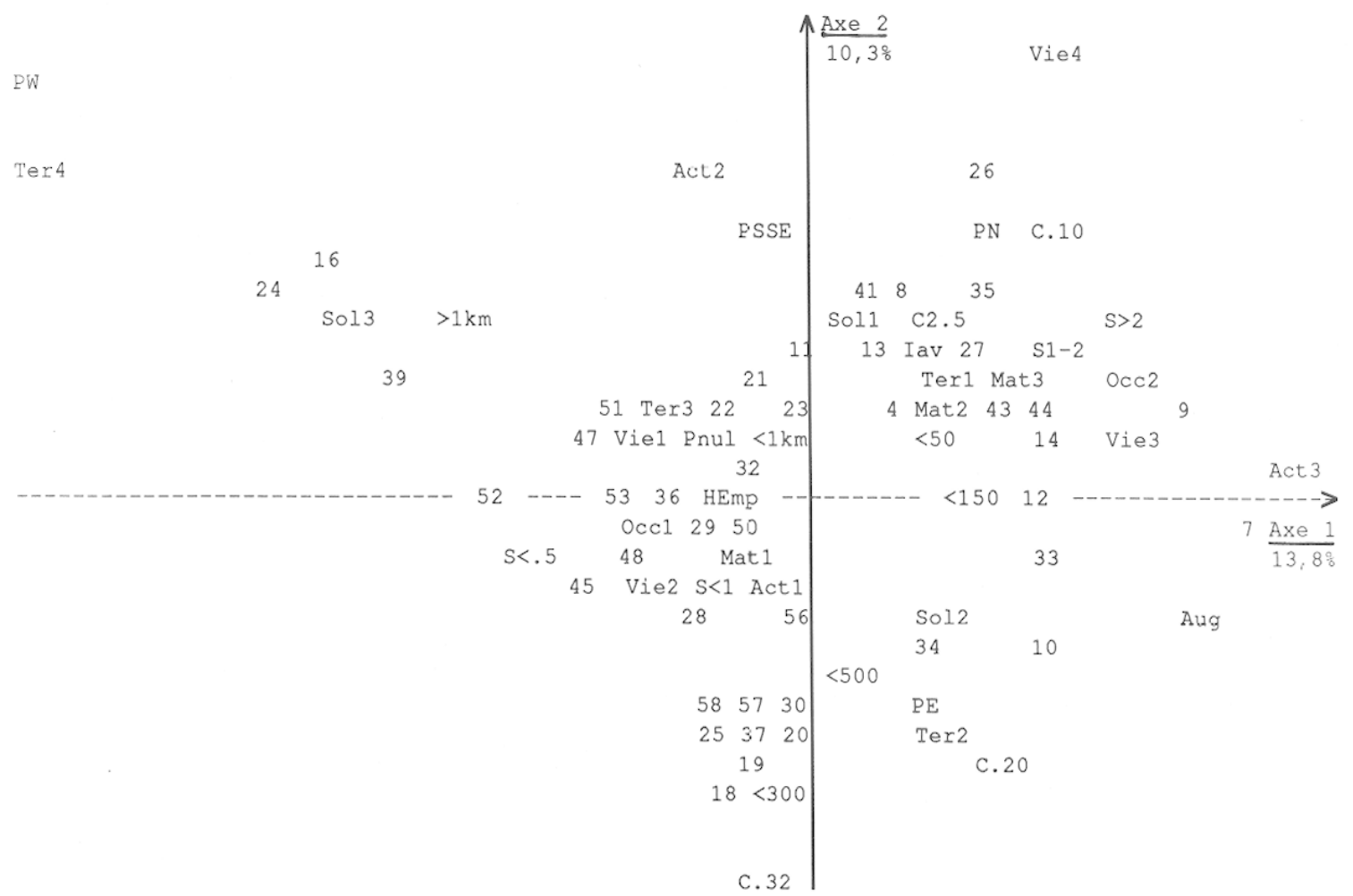

Fig. 4 - Analyse factorielle des correspondances avec les données paysagères (plan 1-2).

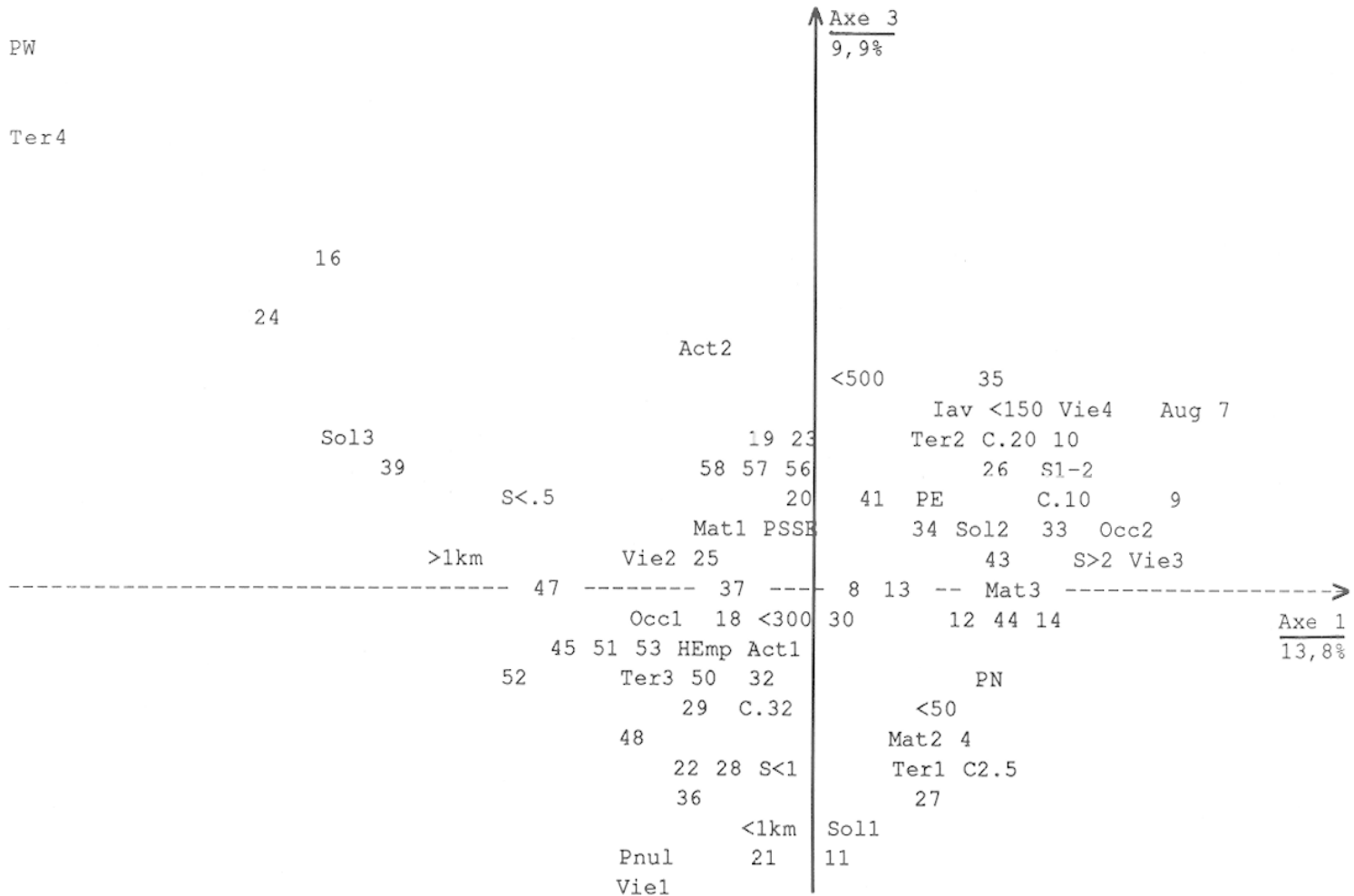

Fig. 5 - Analyse factorielle des correspondances avec les données paysagères (plan 1-3). 
2 (mobilier d'artisanat, outillage agricole), regroupant à la fois des sites de garrigue et des sites installés sur les autres terroirs; à l'opposé, en bas du graphe, on repère les sites à simple activité de stockage (amphore, dolium : Act. 1).

\section{L'AXE 3 (fig. 5)}

Il structure le groupe des sites de plaine et de coteau, généralement caractérisés par le niveau d'activité 1 : il différencie les sites de plaine sur sols limoneux, regroupés vers le haut, des sites de cuesta sur sols mixtes, localisés en bas du graphe.

\section{PREMIERE CLASSIFICATION ASCENDANTE HIERARCHIQUE (CAH)}

Une première classification (fig. 6) a été réalisée avec le logiciel DATAVISION ${ }^{12}$ : l'examen de l'arbre obtenu à l'issue des calculs permet d'affiner l'analyse des graphes de l'AFC. Les six descripteurs retenus pour cette analyse ont été limités aux seules informations liées spécifiquement au matériel (superficie, matériaux, mode de vie, activité, durée d'occupation, date d'implantation), complétés par un unique caractère paysager, le terroir, c'est-à-dire les caractères qui apparaissaient comme les plus structurants dans l'AFC. En outre, dans cette première approche, on a choisi de ne classer que les sites.

\section{Structure}

\section{CLASSE I}

La première classe (fig. 7) ne réunit que des sites à niveau de vie $1(100 \%$ des Vie 1$)$ et $2(92 \%$ des Vie $2)$; ce sont, à trois exceptions près ( $\mathrm{n}^{\mathrm{os}} 44,41$, et 30 ) des sites n'ayant livré que du matériel du HautEmpire : parmi eux, le site 19, daté de l'Antiquité tardive. Cette occupation restreinte est renforcée par le fait qu'ils fonctionnent tous à partir du HautEmpire, sauf les sites 16,56 et 44 , qui ont fourni de l'arétine de l'époque augustéenne. Ce sont, en général, des sites à matériel de niveau 1 : sauf les sites 53 et 21 , de niveau 2 , et les sites $56,51,50,11$ et 13 , de niveau 3.

La première différenciation au sein de la classe est opérée entre : le groupe 1.1 des sites de garrigue et de cuesta (des $n^{\text {os }} 39$ à 18 : exception notable, le

12 Créé par M. Roux : Algorithmes de classification, Masson, Paris, 1985. site 47 , site de costière) et le groupe I. 2 des sites de plaine et de costière.

I.1 - Groupe des sites de garrigue et de cuesta. En son sein, se différencient les sites de garrigue (I.1a) à sol calcaire $\left(n^{\circ} 3\right)$ auxquels se rattache le site de costière 47 , à sol de niveau 2 , mais qui partage le caractère Act. 2 avec deux des trois sites de garrigue (voir l'axe 2, fig. 4); les sites de cuesta ( $I .1 b^{1}$ et $b^{2}$ ) subdivisés entre les sites de superficie d'épandage $<0,5$ ha $\left(\mathrm{n}^{\text {os }} 58,25,57,37,19,56\right)$, et ceux de superficie $<1$ ha $\left(\mathrm{n}^{\circ \mathrm{s}} 20,18\right)$. Ce sont tous des sites à activité de niveau 1 (stockage), qui se différencient donc du groupe I.la de ce point de vue (sauf le $n^{\circ} 39$, Act. 1), et à niveau de vie 2, comme le groupe I.1a.

I.2 - Groupe des sites de plaine el de costière. Il est réparti, dans une prcmic̀rc ćtape, cntrc d'unc part : les sites I.2a de superficie $<0,5$ ha $\left(1.2 \mathrm{a}^{1}\right.$ : $\left.\mathrm{n}^{\text {os }} 48,45,52,51,50\right)$ regroupés contradictoirement avec les sites possédant la modalité opposée, soit $>2$ ha $\left(1.2 \mathrm{a}^{2}: \mathrm{n}^{\mathrm{os}} 44,41,11,13\right)$; et d'autre part les sites I. $2 \mathrm{~b}<1$ ha $\left(\mathrm{n}^{\text {os }} 36,28,29,22,32,30,53,21\right)$. Dans ce groupe, les sites se subdivisent entre les sites à Mat. 1 (des $\mathrm{n}^{\text {os }} 36$ à 30$)$ et ceux à Mat. 2 (n $\mathrm{n}^{\text {os }} 53$ et 21 ).

Si le groupe I.2 ne comporte pas de division matérialisée entre les sites de costière et ceux de plaine, on note un regroupement significatif des sites de costière entre les sites 48 et 41 , et des sites de plaine entre les $\mathrm{n}^{\text {os }} 11$ et 21 , le site de costière 53 s'intercalant dans ce sous-ensemble pour se rapprocher du site 21, Mat. 2 comme lui.

\section{CLASSE II}

La seconde classe, qualitativement la plus élevée (des sites $34,27,4$ à 7 ) ne réunit, à l'exception des sites 12 et 23 qualifiés Vie 2 , que des sites appartenant aux catégories Vie 3 et Vie 4 : ce sont tous des sites de plaine et de cuesta, sauf le 43 , site de costière.

A deux exceptions près (sites 23 et 12), ils ont une durée d'occupation de niveau 2 (Haut et BasEmpire), renforcée par le fait que cinq d'entre eux (plus de $35 \%$ ) fonctionnent dès le $\mathrm{i}^{\text {er }} \mathrm{s}$. av. J.-C. $\left(\mathrm{n}^{\text {os }} 34,14,23,26,09\right)$, et quatre (plus de $28 \%$ ) dès l'époque augustéenne ( $\left.\mathrm{n}^{\text {os }} 10,43,12,7\right)$.

La structuration de cette classe se fonde sur les trois points suivants: la différenciation entre le groupe le plus élevé II.2, celui qui fournit des indices d'installations spécialisées (fours, pressoirs, celliers), soit Act. 3 ( $\mathrm{n}^{\text {os }} 12,9,7$ ) ; et deux autres groupes, euxmêmes différenciés selon le même critère : II.lb le groupe Act. 2 (outillage et mobilier à caractère 


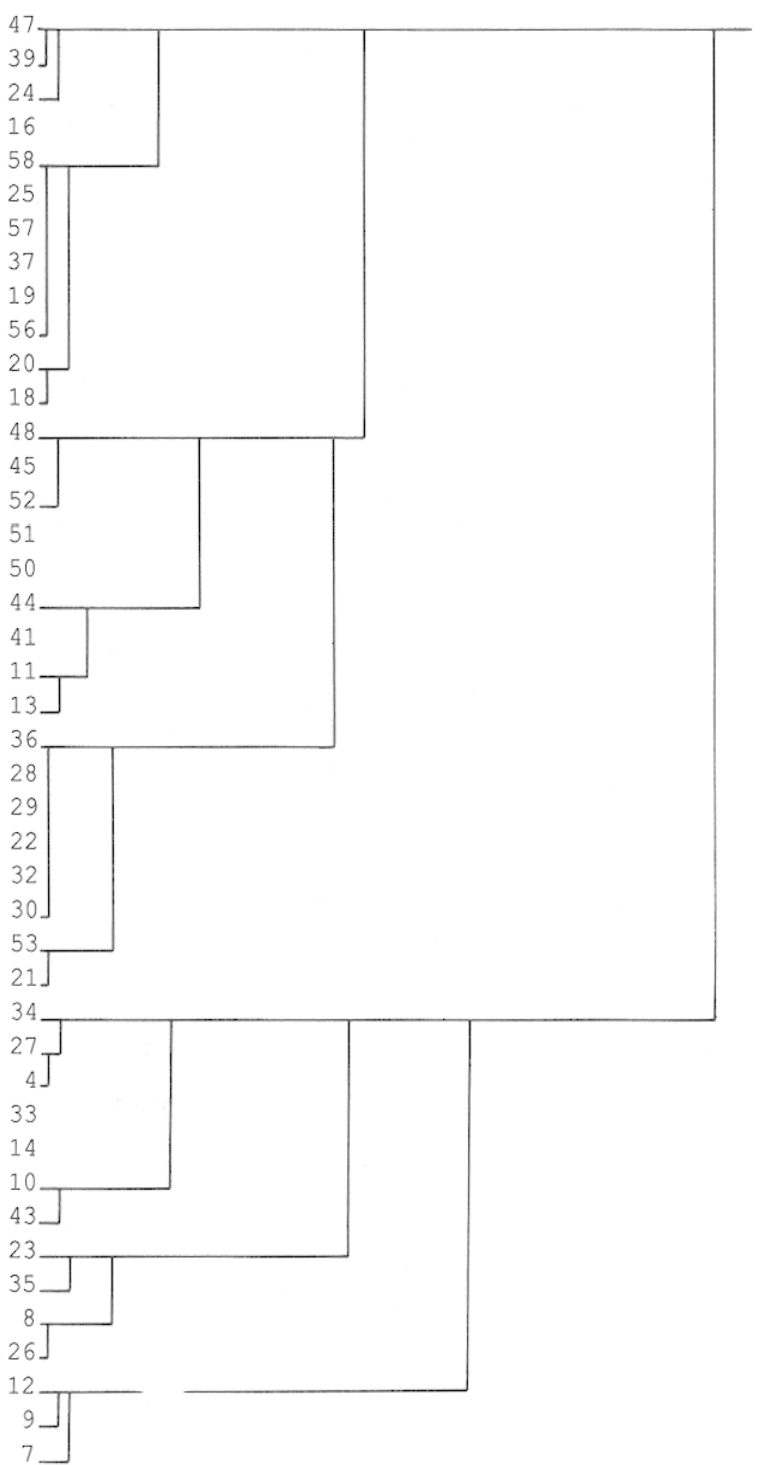

Fig. 6 - Classification ascendante hiérarchique des sites sans les données paysagères.

artisanal et agricole', : sites $23,35,8,26$; et II.1a le groupe Act. 1 (activité de stockage) : sites $34,27,4,33,14,10,43$; ce dernier groupe de la seconde classe ne réunit que des Vie 3 . Le reste des deux autres groupes est Vie 4, sauf les sites 23 et 12 .

La surface d'épandage des matériaux et mobiliers ne constitue pas un réel facteur de structuration de cette seconde classe, encore que les sites allant du $\mathrm{n}^{\mathrm{0}} 10$ au 7 soient tous supérieurs à 1 ha (S $1-2$ des $\mathrm{n}^{\text {os }} 10$ à 35 , et $\mathrm{S}>2$ des $\mathrm{n}^{\text {os }} 26$ à 7 ), à l'exception de l'anomalie du site $8(\mathrm{~S}<0,5)$ qui constitue sans

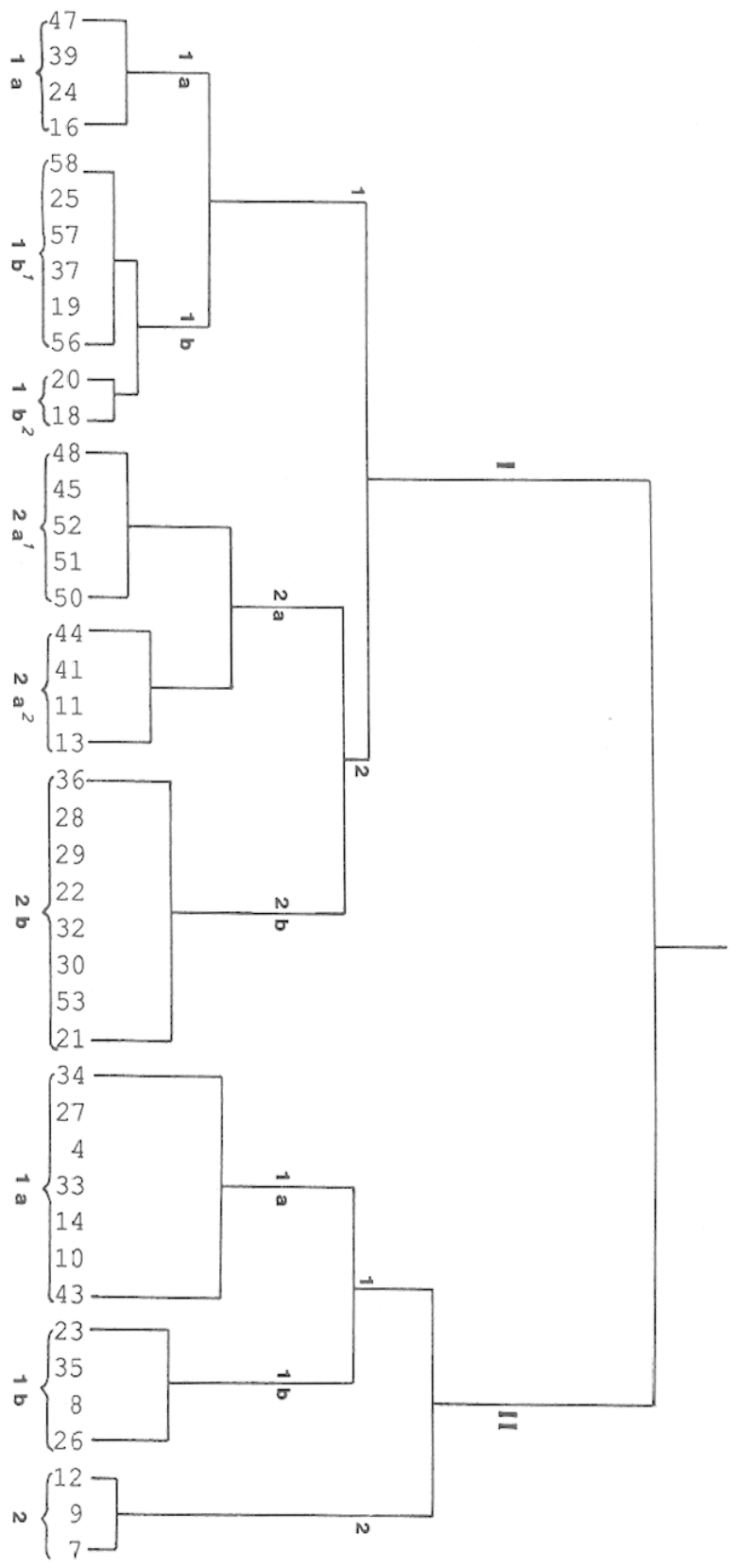

Fig. 7 - Dendrogramme à interprétation des données.

doute un faux petit site ${ }^{13}$. Entre les sites 34 et 14, la situation est plus confuse de ce point de vue. De plus, on notera, dans la classe II.2, la différenciation entre le groupe 9 et 7 (Mat. 3, Vie 4, Occ. 2) et le site 12 (Mat. 2, Vie 2, Occ. 1).

13 Il s'agit, en effet, du site de Saint-Pierre-deCampublic qui, avant de connaître un développement remarquable au Moyen Age, constituait, au $\mathrm{XI}^{\mathrm{e}}$ s., une villa, propriété d'un comte de Charlemagne puis de l'archevêque d'Arles. 


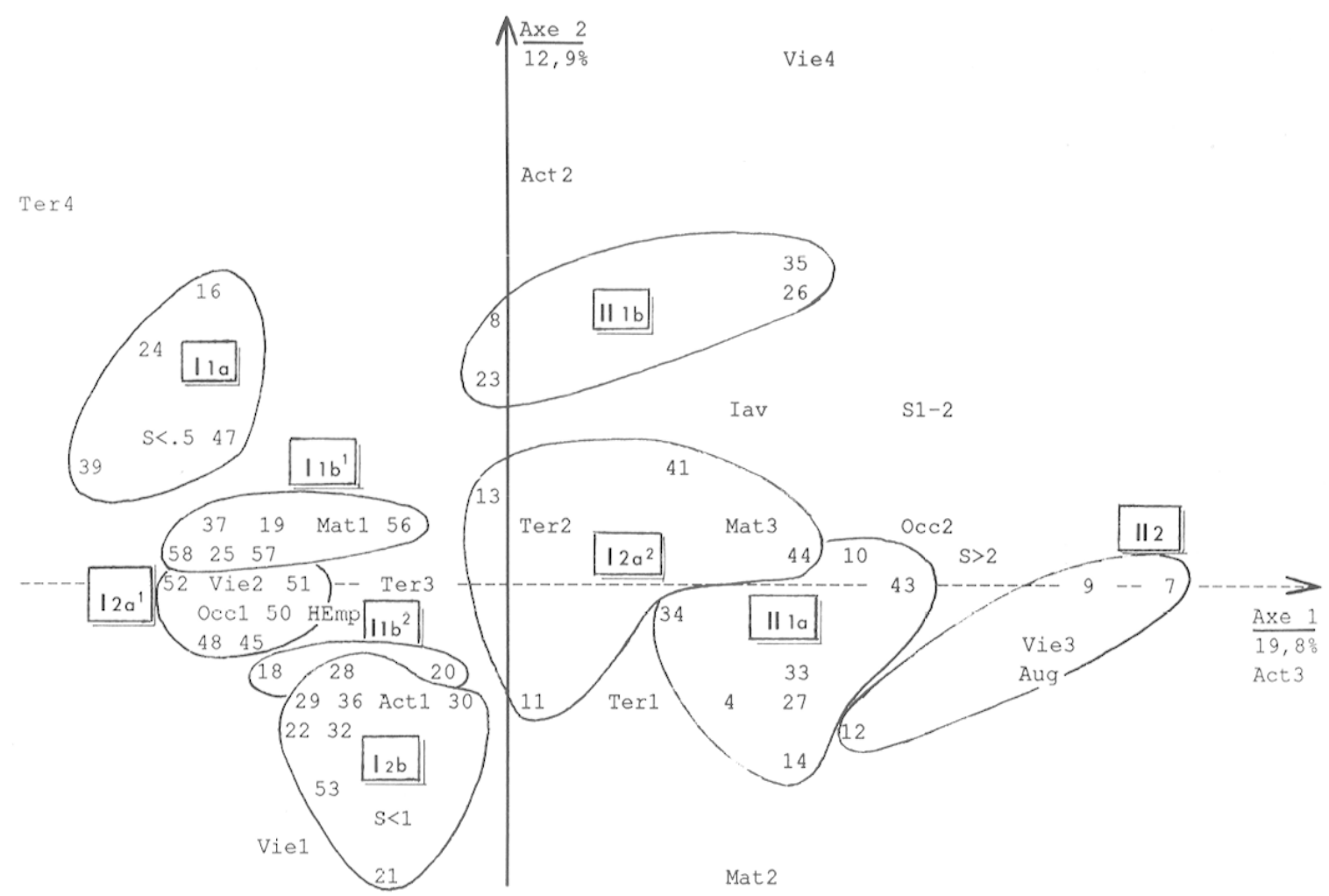

Fig. 8 - Analyse factorielle des correspondances et regroupement des sites sans les données paysagères.

\section{RÉsultats}

La structure linéaire de l'arbre a facilité l'analyse et la compréhension du mode d'organisation spatiale des ensembles de sites et de caractères révélée par les graphes de l'AFG. Toutefois, ce découpage en classes selon un ordre hiérarchique gomme les liaisons, les connexions ou les antinomies, mises en évidence par la distribution spatiale des groupes d'individus et des caractères dans chacun des graphes.

Du point de vue général de l'arbre, on constate que dans la seconde classe, la plus élevée, ce sont le niveau d'activité et le niveau de vie qui structurent les groupes de sites. On trouve, dans cette classe, des sites à implantation précoce et à durée d'occupation relativement longue. Ce sont, en général, des sites de grande superficie. L'examen des autres caractères, non pris en compte dans cette classification, montre qu'ils sont relativement proches des grandes voies de communication, et qu'ils s'inscrivent dans des parcellaires isoclines avec les cadastres antiques régionaux : le $\mathrm{A}$ affiché à Orange et le $\mathrm{B}$ de Nîmes.

Dans la première classe, où dominent les sites à Mat. 1 (76\% d'entre eux), à simple activité de stockage (près de $80 \%$ ), à niveau de vie $1(13 \%$ ) et 2 (près de $80 \%$ ), ce sont successivement les terroirs et les superficies d'épandage qui différencient les groupes de sites, où l'originalité de certains individus peut s'exprimer dans le niveau d'activité (voir le comportement des Act. 2) et la nature du matériel livré : Mat. 2 ou 3. Ce sont généralement des sites implantés au Haut-Empire, voire au Bas-Empire $\left(\mathrm{n}^{\circ} 19\right)$, et de durée d'occupation relativement restreinte; à plus de $85 \%$, ils ont une superficie d'épandage inférieure à 1 ha et à $52 \%$ inférieure à 0,5 ha. On sait par ailleurs que plus de $40 \%$ sont implantés à plus d' $1 \mathrm{~km}$ des grandes voies de communication. Les sites inscrits dans un parcellaire induit par les deux cadastres évoqués plus haut ne se rencontrent qu'en plaine et sur la costière $\left(\mathrm{n}^{\text {os }} 37\right.$, $44,41,11,13,22,21)$.

L'arbre peut être mis en rapport, dans son développement, avec l'organisation spatiale des sites sur les graphes produits par ANACONDA, en éclairant la structure du groupe situé au centre des graphes, c'est-à-dire entre le groupe des sites de garrigue, bien individualisé à un extrême, et celui des sites de la seconde classe, à l'autre extrême (fig. 8). 


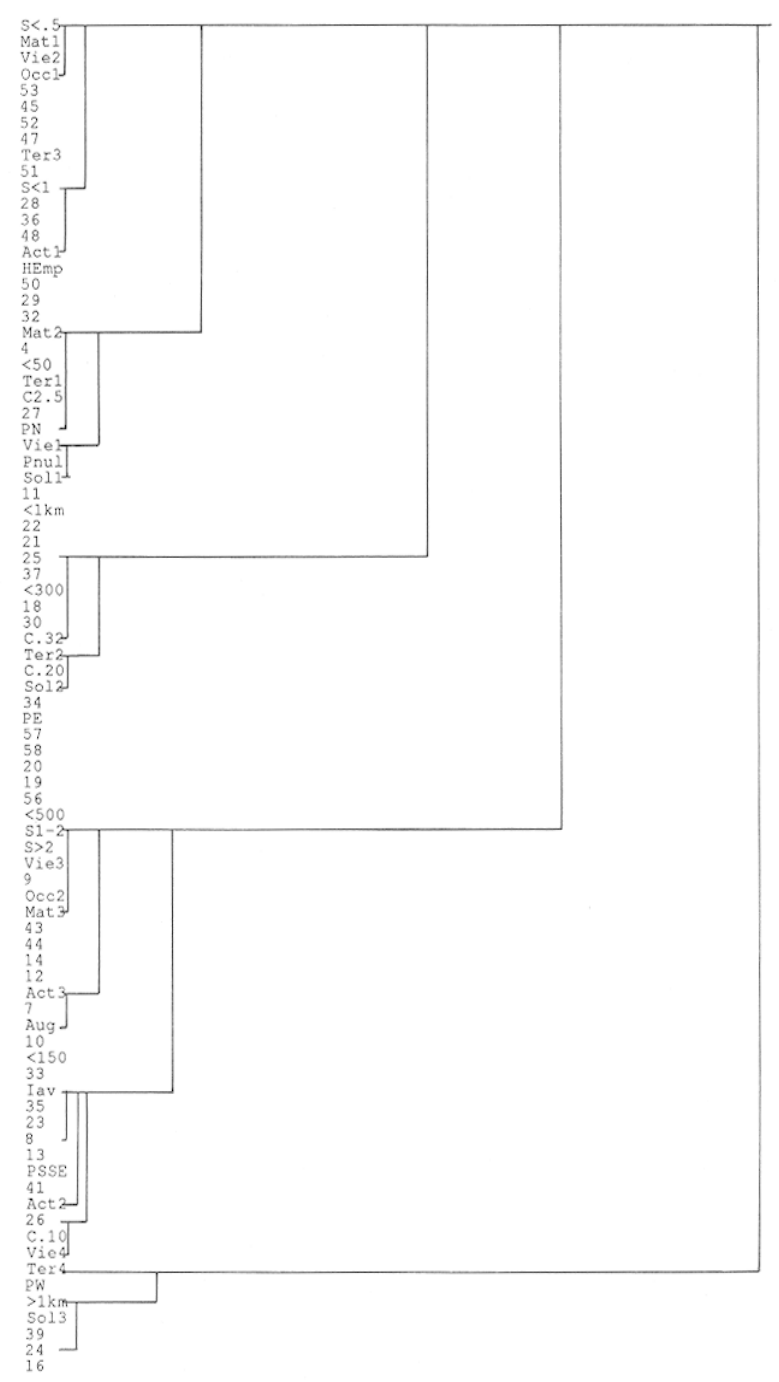

Fig. 9 - Classification ascendante hiérarchique des sites avec les données paysagères.

\section{SECONDE CLASSIFICATION ASCENDANTE HIÉRARCHIQUE}

A la suite de cette première expérience, nous avons directement soumis les résultats de l'analyse factorielle des correspondances à une nouvelle classification, avec le concours d'un logiciel élaboré, à partir des algorithmes de M. Roux, par J.-J. Girardot ${ }^{14}$. Dans ce cas, la classification intègre à la fois les sites et leurs descripteurs (fig. 9 et 10), chacun des caractères se trouvant localisé au cœur des groupes de sites qui le partagent le plus. D'autre part la classification s'opère à partir des distances

14 Logiciel ANCAH, qui fonctionne actuellement en version expérimentale.

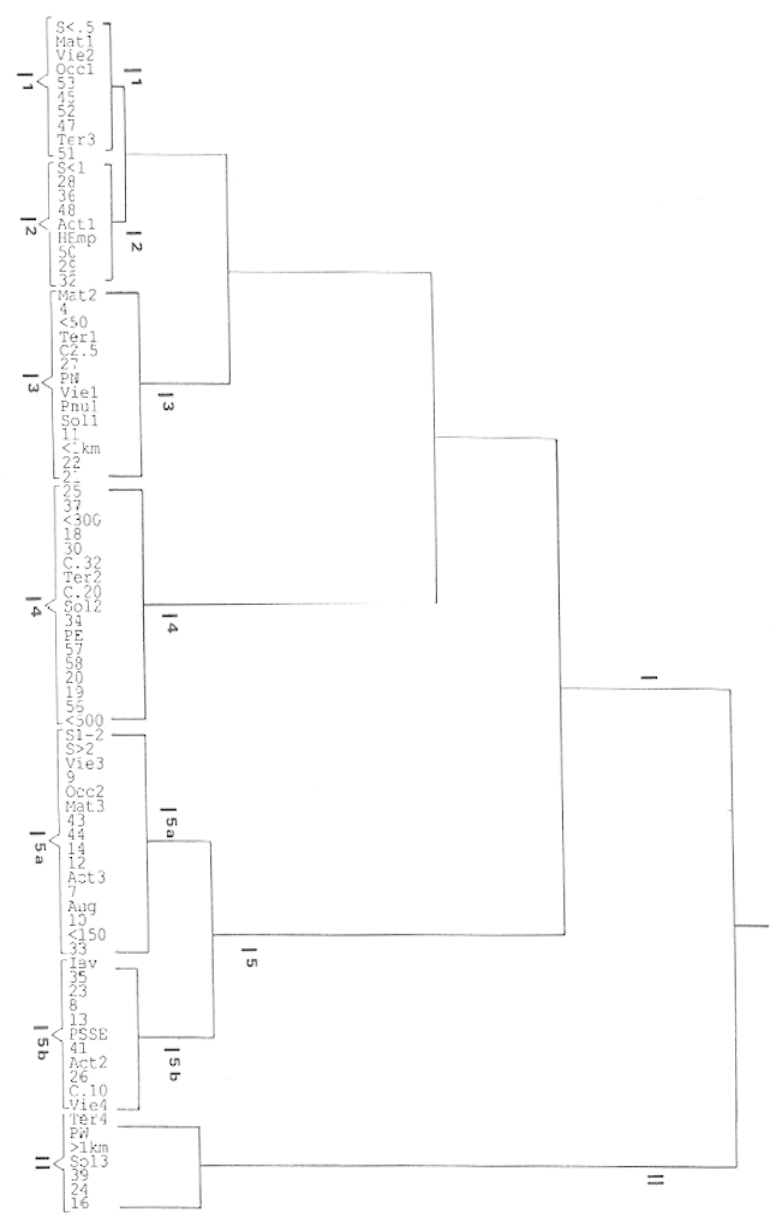

Fig. 10 - Dendrogramme à interprétation des données.

entre les points tels qu'ils sont disposés dans l'espace factoriel : les classes peuvent être directement reportées sur le graphique. A cette étape du travail, nous avons choisi de retenir tous les descripteurs pour étudier l'impact des données paysagères sur la typologie des sites, et pour mieux comparer les résultats de la classification et l'information donnée par les graphes de l'AFC (fig. 11, indiquant les groupes de sites).

\section{Structure}

Dans ce cas, la seconde classe rassemble les seuls sites de garrigue ( $\left.\mathrm{n}^{\text {os }} 39,24,16\right)$, et cette nouvelle classification montre clairement une division de la première classe en six groupes :

1. Le groupe $I .1$ ( $\mathrm{n}^{\mathrm{os}} 53$ à 51$)$ rassemble cinq sites de costière, de dimensions modestes $(\mathrm{S}<0,5 \mathrm{ha}$ ) et 


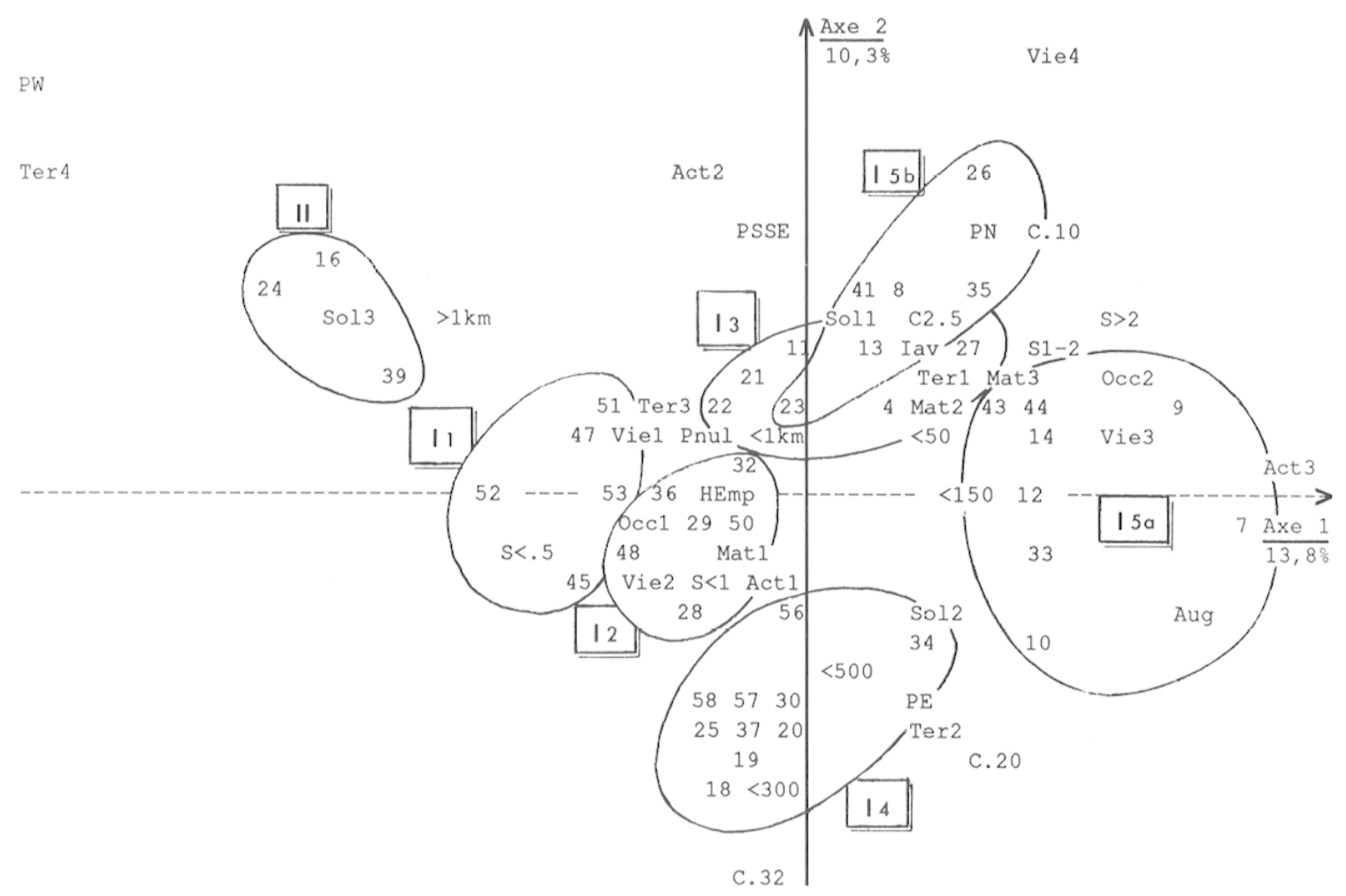

Fig. 11 - Regroupement des sites selon l'AFC avec les données paysagères.

de niveau de vie 2 (sauf le 45 , Vie 1). Ici, le site 47 , rattaché dans la classification $M$. Roux au groupe de la garrigue par le caractère Act. 2, est intégré dans le groupe de la costière (tous Occ. 1 et Haut-Empire).

2. Le groupe $1.2 \quad\left(\mathrm{n}^{\text {os }} 28\right.$ à 32$)$ délimite un ensemble mixte de petits sites de plaine $(\mathrm{S}<1$ ha) et de costière ( $\mathrm{S}<0,5 \mathrm{ha}$ ), de matériel 1 (sauf le 50 , Mat. 3) et de niveau de vie 2 (sauf le 48 , Vie 1) : ils partagent tous Act. 1, Occ. 1 et Haut-Empire. Le seul caractère qui justifie l'exclusion des sites 48 et 50 de leur groupe d'origine (celui de la costière) est celui de la distance par rapport à la voirie majeure $(<50 \mathrm{~m})$, qu'ils partagent avec le site 36 , voire avec le $32(<150 \mathrm{~m})$ : en revanche les sites de costière du groupe précédent sont, pour l'un, à plus de $150 \mathrm{~m}$ et, pour le reste, à plus de $500 \mathrm{~m}$ des voies de communication. Dans ce cas, les critères paysagers semblent faire la décision dans la typologie.

3. Le groupe $I .3$ ( $\mathrm{n}^{\text {os }} 4$ à 21) réunit cinq sites de plaine, sur Sol 1, qui présentent une certaine diversité au plan des superficies, de la nature du matériel collecté et du niveau de vie qu'il exprime: en revanche, ils sont tous Act. 1, Haut-Empire et tous s'intègrent dans un réseau de limites agraires orientées à $\mathrm{N} 2^{\circ} 5^{\prime} \mathrm{E}$. Dans ce cas également, une donnée paysagère réussit apparemment à structurer un groupe que les critères tirés du matériel, si importants par ailleurs, ne pouvaient suffire à unifier.

4. Le groupe $I .4$ ( $\mathrm{n}^{\mathrm{os}} 25$ à 56 ) regroupe tous les sites de cuesta inférieurs à 1 ha de superficie : s'y ajoute un site de plaine, également de $S<1$ ha, et qui a la particularité de partager avec ses compagnons de groupe le caractère Sol 2, original pour un site de plaine, et le caractère PE. En outre, tous ces sites sont implantés à une distance comprise entre 150 et $500 \mathrm{~m}$ des grandes voies de communication. En ce qui concerne le matériel, ils sont généralement à $90 \%$ : Mat. 1 isauf le 56 , Mat. 3 ) - Vie 2 (sauf le 34 , Vie 3) - Act. 1 (sauf le 37, pour lequel le caractère fait défaut).

5. Le groupe I.5a ( $\mathrm{n}^{\text {os }} 9$ à 33$)$ réunit les gros sites de plaine, coteau et costière, supérieure à 1 ha d'épandage, à l'exception du site $14(<1 \mathrm{ha})$. Hormis le site 33 (Haut-Empire), ils sont tous implantés à une époque précoce, dès le $\mathrm{I}^{\mathrm{er}} \mathrm{s}$. av. J.-C. ou à la période augustéenne, et sont occupés jusqu'au BasEmpire (à l'exception du site 12). Ce qui structure le groupe, c'est le niveau de vie (Vie 3, sauf le site 12) : ce dernier trouve sa place au sein du groupe grâce, entre autres, au caractère Act. 3 qu'il partage avec les sites 7 et 9 . Tous ces sites sont localisés à moins de $300 \mathrm{~m}$ des grandes voies de communication. 


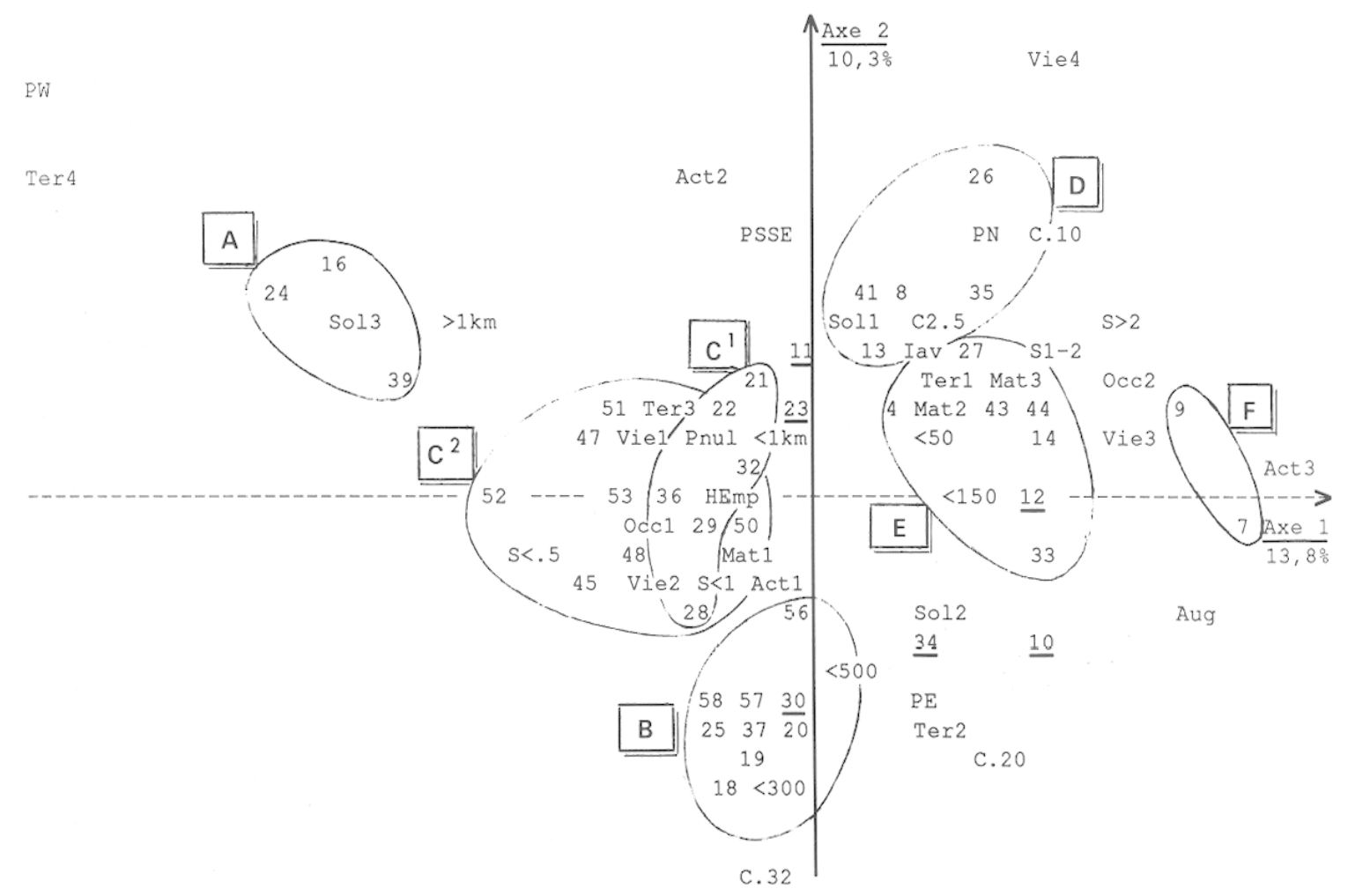

Fig. 12 - Synthèse de l'analyse des données.

6. Le groupe $I .5 b\left(\mathrm{n}^{\circ \mathrm{s}} 35\right.$ à 26$)$ : il nous a paru instructif de dissocier un sixième groupe du groupe précédent, dans la mesure où l'arbre opère une division secondaire au sein de ce groupe de sites importants soit par la superficie, soit par la richesse de leur matériel, et généralement par la combinaison des deux facteurs.

En effet, ce dernier groupe réunit également des sites supérieurs à 1 ha (à l'exception du $n^{\circ} 8$, $\mathrm{S}<0,5$ ha), mais plutôt d'implantation relativement tardive en regard du groupe précédent (deux sites cependant datent du $\mathrm{I}^{\mathrm{er}} \mathrm{s}$. av. J.-C.). Le niveau de vie ne descend jamais au-dessous du degré 2 ; mais ce qui structure le plus remarquablement ce groupe, c'est bien le caractère Act. '2.

\section{SYNTIIÈSE}

LES GROUPES

Au terme de l'analyse des données, il est possible de distinguer deux classes de sites, divisées chacune en trois groupes (fig. 12 et 13 ).

\section{CLASSE I}

Elle rassemble les sites à Mat. 1, à simple activité de stockage el à niveau de vie 1 ou 2 .
Ce sont des sites dont l'occupation ne se prolonge pas dans l'Antiquité tardive à l'exception du $n^{\circ} 19$, occupé seulement au Bas-Empire : rares sont ceux qui ont livré du matériel du $\mathrm{I}^{\mathrm{er}} \mathrm{s}$. av. J.-C. $\left(n^{\text {os }} 21,22\right)$ ou d'époque augustéenne $\left(n^{\circ} 20\right)$. Peu de sites de cette classe s'insèrent dans un parcellaire induit par les deux cadastres antiques.

Les groupes de cette classe sont différenciés successivement par les terroirs et par les superficies d'épandage qui ne dépassent pas 1 ha.

Groupe $A$. Au nombre de trois (n ${ }^{o s} 39,24,16$ ), les sites de garrigue sur sol calcaire sont de faible superficie $(<0,5 \mathrm{ha})$, mais se distinguent aussi par un fort éloignement par rapport aux voies de communication $(>1 \mathrm{~km})$, et par la prédominance du caractère Act. 2. Ce sont des établissements modestes, que l'on peut supposer indépendants ou liés à des habitats plus importants, implantés notamment dans le bassin de Jonquières.

Groupe B. Ces huit sites de cuesta (n ${ }^{\text {os }} 25,37,18$, $57,58,20,19,56)$ présentent, pour la plupart, une superficie inférieure à 0,5 ha : deux atteignent cependant 0,7 et 1 ha ( $n^{\text {os }} 20$ et 18 ). Ils se situent généralement à moins de $300 \mathrm{~m}$ d'une route (à l'exception des $n^{\text {ss }} 19$ et 20 à $\left.<500 \mathrm{~m}\right)$. Dans ce groupe, le site 56 se singularise par son matériel (Mat. 3) et une occupation plus ancienne, dès le $\mathrm{I}^{\mathrm{er}} \mathrm{s}$. 


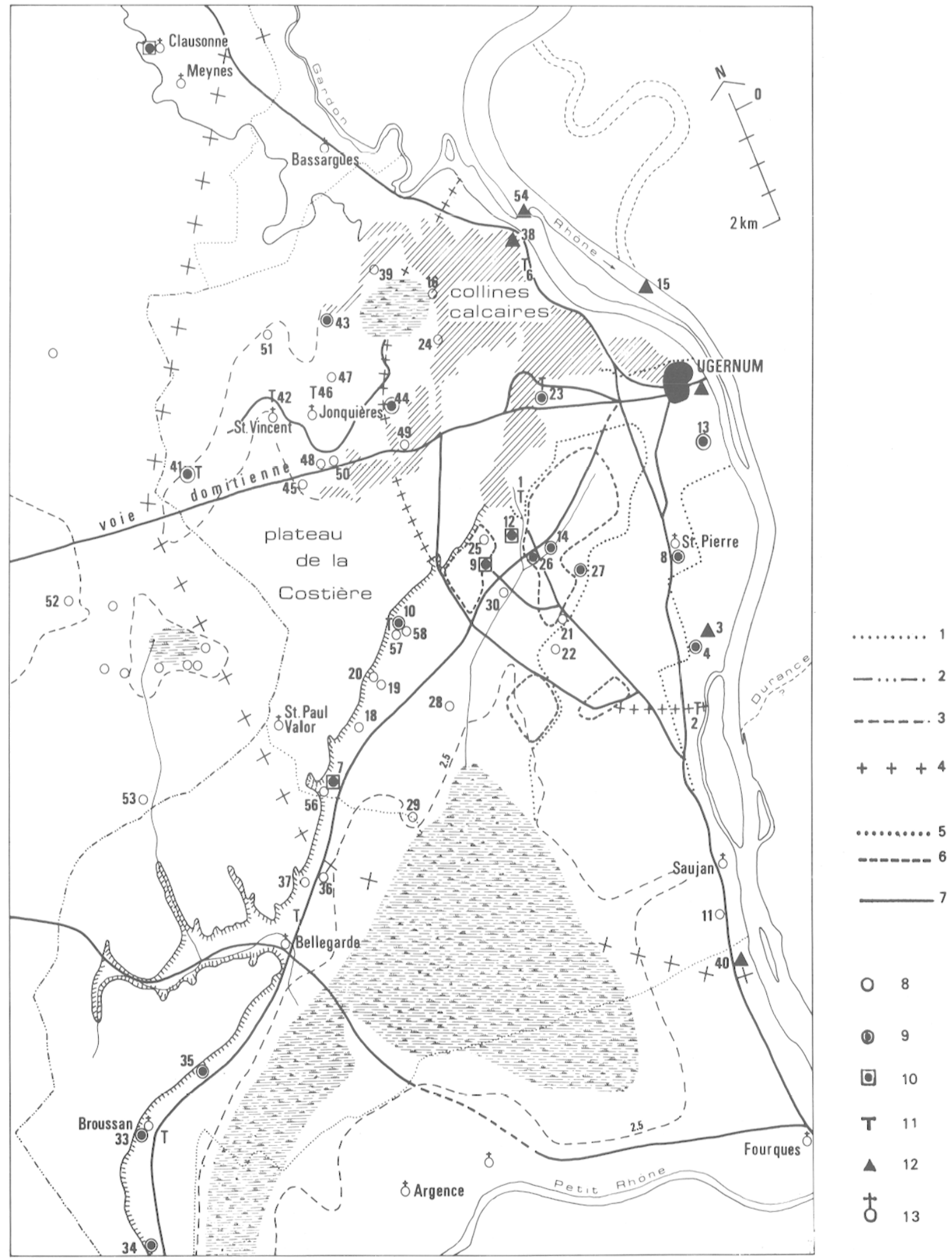

Fig. 13. - Le Beaucairois : Limites, réseaux et implantation des sites.

Limites et réseaux : 1 , limite de commune; 2 , limite de canton; 3 , limite de dépressions mal drainées; 4 , limite des 5 et $10 \mathrm{~km}$ à partir de la ville. - Zone d'imbrication des cadastres ruraux antiques dans la plaine : 5 , réseau Orange A; 6 , réseau Nîmes $B ; 7$, réseau routier antique.

Implantation des siles : 8, habitats gallo-romains de la classe I (groupes A-C); 9 , habitats gallo-romains de la classe II (groupes D-E) ; 10, habitats gallo-romains de la classe II (groupe F); 11, tombes d'époque gallo-romaine; 12, monuments d'époque gallo-romaine; 13 , paroisses médiévales. 
av. J.-C. Certains se placent comme des dépendances au contact d'établissements plus vastes ( $\mathrm{n}^{\circ} 56$ par rapport à $7 ; n^{0 s} 57$ et 58 par rapport à 10 ) ou à faible distance $\left(\mathrm{n}^{\text {os }} 25\right.$ et 9$)$; d'autres s'intercalent de façon régulière entre les sites plus gros et n'ont pas forcément une nature différente de ces derniers.

Groupe $C$. Dans cet ensemble mixte de sept sites de costière $\left(\mathrm{n}^{\mathrm{os}} 45,47,48,50,51,52,53\right)$ et six de plaine $\left(\mathrm{n}^{\text {os }} 21,22,28,29,32,36\right)$, la superficie d'épandage permet de distinguer deux sous-groupes : en effet, les sites de plaine (C.1) ont une surface comprise entre 0,5 et $1 \mathrm{ha}$, alors que les sites de costière (C. 2$)$ ne dépassent pas 0,5 ha, à l'exception $\mathrm{du} \mathrm{n}^{0} 53$.

Si leur rapport à la voirie est variable, la majorité est localisée à plus de $300 \mathrm{~m}$ des routes. Dans ce groupe, plusieurs individus se distinguent par la nature du matériel (Mat. 2 pour les $n^{\text {os }} 21$ et 53 ; Mat. 3 pour le 51) qui peut indiquer la présence d'une villa.

Les autres sites de costière ne se distinguent guère des fermes ou des dépendances de la garrigue (sauf pour l'activité). Mais ce groupe est surtout caractérisé par la situation de la plupart de ses individus dans la partie basse de la plaine (entre 2,5 et $3 \mathrm{~m}$ d'altitude) ou dans la dépression de Jonquières. On y verra donc essentiellement des établissements de type coloniaire, liés à la mise en valeur des terrains lourds qu'il était nécessaire de drainer.

\section{CLASSE II}

Dans la seconde classe, ce sont le niveau d'activité et le mode de vie (Vie 3 ou 4, à l'exception des sites n ${ }^{\text {os }} 12$ et 13) qui structurent les groupes de sites. Les sites à Mat. 1 y sont minoritaires (quatre sur quinze).

Cette classe comporte des sites à implantation précoce et à durée d'occupation relativement longue. Ce sont généralement des sites de grande superficie (mais ce n'est pas systématique: un tiers sont inférieurs à $1 \mathrm{ha}$ ), le plus souvent proches des routes et inscrits dans des parcellaires isoclines avec les cadastres romains. La répartition selon les terroirs est moins nette que dans la première classe : on rencontre ici la majorité des sites de plaine, aucun n'est localisé en garrigue.

Groupe D. Ce groupe mixte de cinq sites $\left(\mathrm{n}^{\mathrm{os}} 8\right.$, $13,26,35,41$ ) est caractérisé par le niveau d'activité 2. Il rassemble tous les sites de mode de vie 4 . On y observe, dans trois cas, une opposition Mat.1/ Vie 3 ou 4. Ces sites, de longue durée, n'ont, à l'exception du n" 26 , livré aucun matériel antérieur au Haut-Empire. Le ${ }^{\circ} 13$ se singularise par un niveau de vie 2 et une occupation limitée au HautEmpire.

Groupe E. Les six sites de cette classe (n ${ }^{0 \mathrm{~s}} 4,14$, 27, 33, 43, 44) partagent le caractère Act. 1. Leur's matériaux sont de classe 3 et surtout 2 , à l'exception du $n^{\circ} 44$ (Mat. 1), mais révèlent pour tous un mode de vie de niveau 3 . Ils sont occupés durant tout l'Empire, mais la moitié a livré un matériel plus ancien ( $\mathrm{I}^{\text {er }}$ s. av. J.-C. ou augustéen).

Les sites de plaines $n^{\text {os }} 4$ et 27 , pleinement intégrés dans ce groupe, mixte au plan des terroirs, par les caractères Mat. 2 ou 3, Act. 1, Vie 3 et Occ. 2, tendent toutefois à être attirés (fig. 4) vers le sousgroupe C. 1, dont ils partagent le terroir et le type de sol, l'implantation au Haut-Empire et l'activité de stockage : en outre, ils s'incrivent, comme trois autres membres de ce même sous-groupe, dans un parcellaire isocline avec le cadastre A d'Orange (C. $\left.2^{\circ} 30^{\prime}\right)$.

Groupe $F$. Ce groupe, limité à deux individus $\left(n^{\text {os }} 7\right.$ et 9$)$, correspond aux sites majeurs de cette série. Ils ont en commun une superficie supérieure à 2 ha, les caractères Mat. 3, Vie 3 et surtout Act. 3, indice d'installations spécialisées. L'un a livré des témoins $d u \mathrm{r}^{\mathrm{er}} \mathrm{s}$. av. J.-C., l'autre d'époque augustéenne. L'un est localisé en plaine, à proximité de la cuesta, l'autre est au piedmont même.

Les sites de cette classe forment l'essentiel du tissu des villae du territoire, même si certaines sont comprises dans la classe I, notamment dans le groupe C. D'ailleurs, les trois groupes de cette seconde classe illustrent vraisemblablement une hiérarchie; il semble en effet que l'on puisse restituer un domaine de 50 ha environ à la villa $\mathrm{n}^{\circ} 26$ (groupe D), mais plus de 100 ha à la villa $\mathrm{n}^{\circ} 9$ (groupe F) ${ }^{15}$.

\section{LES INCLASSABLES}

L'intérêt de l'analyse factorielle n'est pas seulement de fournir une aide à la définition de groupes, mais aussi d'attirer l'attention sur des individus qui échappent au fonctionnement de la typologie.

Deux d'entre eux apparaissent au sein de groupes dont ils ne partagent pas pour autant la définition générale : le $\mathrm{n}^{\circ} 30$, site de plaine parmi un groupe de sites de cuesta (B), et le $\mathbf{n}^{0} 12$, gros site de plaine de niveau de vie 2 et d'occupation de

15 Développement dans Etudes sur Ugernum ..., op. cit., chapitre 3 . 
catégorie 1, au sein d'un groupe de niveau 3 et d'occupation de catégorie 2 (E-F). La plupart se placent, sur les graphes, à la limite des deux classes détaillées plus haut: $\mathrm{n}^{\text {os }} 10,11,23$ et 34 . Pour certains, il s'agit seulement d'une position marginale entre deux groupes qui peuvent les intégrer. Pour d'autres, on ne peut exclure, comme on va le voir, une nature singulière, grosse de conséquences pour leur insertion dans la typologie.

Dans le cas de simple marginalité, on peut distinguer les cas limites inter-groupes ( $\mathrm{n}^{\mathrm{os}} 30$ et 12 ) et inter-classes $\left(n^{\circ} 11\right.$ et, sans doute, $\left.n^{\circ} 10\right)$. Dans la classe I, on est d'abord étonné par la position du site de plaine $n^{0} 30$, déjà excentré par rapport aux sites de plaine du groupe C. 1, et intégré au groupe B des sites de cuesta (fig. 12). En réalité, ce site partage des caractères paysagers avec les sites de cuesta (sol 2, pente e. et insertion dans les parcellaires C. 20 et C. 32) qui suffisent à l'éloigner du groupe auquel il est en fait à rattacher : le groupe C. 1 .

De même, dans la classe II, le site 12 se singularise du groupe E, dans lequel il s'insère, pour n'avoir pas livré de témoins de l'Antiquité tardive et par les caractères Mat.2 et Act.3. Ce dernier caractère invite plutôt à l'intégrer au groupe $\mathrm{F}$, dont il n'est pas très caractéristique en raison, peut-être, de prospections insuffisantes.

Quant au site de plaine 11, c'est le cas typique d'un site partagé par des caractères contradictoires entre deux groupes de deux classes différentes. Son occupation courte, son niveau d'activité et son mode de vie l'attirent vers le groupe C. 1 ; ses matériaux, de niveau 3 , et sa grande superficie le rapprochent du groupe E. Comme il s'agit d'un site peu prospecté et encore mal connu, on tendra volontiers à l'intégrer au groupe $\mathrm{E}$.

Le site de cuesta 10 illustre peut-être le même cas de figure : sa vaste superficie, son occupation longue, dès l'époque augustéenne, le rattachent à la seconde classe. Le couple Mat. 1-Vie 3, qui caractérise aussi le $\mathrm{n}^{\circ} 34$, se rencontre, on l'a vu, dans le groupe D. Mais son caractère Act. 1 rejette le $n^{\circ} 10$ hors de ce groupe caractérisé par Act. 2. Les prospections sur ce site ont été importantes; mais les ramassages, qui sont anciens, peuvent avoir négligé les fragments de meules qui signifient généralement le niveau d'activité 2 .

Il est plus délicat de suivre la même démarche pour interpréter la position originale des deux derniers sites 34 et 23 , implantés sur la cuesta. Certes, le site 34 est proche du groupe B, dans lequel on peut songer à l'intégrer. Il s'en distingue cependant par trois traits : un mode de vie 3 , une occupation longue et une implantation précoce; le $n^{0} 56$, déjà excentré dans ce groupe, ne se distinguait des autres que par deux caractères. Le site 34 peut tout aussi bien appartenir à la classe II, mais l'apport de données extérieures à l'analyse peut aider à expliquer sa singularité. Ce site, en effet, implanté aux confins du Beaucairois, est le seul à avoir livré des céramiques du Premier et Deuxième Age du Fer (amphore étrusque, céramique non tournée du Deuxième Age du Fer) : une genèse précoce peut lui avoir conféré une nature et des caractères différents à l'époque romaine.

Quant au site 23 , plus proche que le précédent du groupe $\mathrm{D}$ à cause de son caractère Act. 2 , il s'en distingue par un mode de vie 2 et une occupation qui ne couvre pas le Bas-Empire. En notant que ce site est implanté à la périphérie de la garrigue, on observera que son faciès est proche de celui des sites du groupe $\mathrm{A}$, à l'exception toutefois de sa position par rapport à la voirie - il est en bordure de la Domitienne - et de sa superficie. Ce dernier trait, cependant, est discutable : ce site présente, en effet, la particularité d'offrir sur près de 2 ha des concentrations de tessons d'époque différente; la campanienne s'y trouve, par exemple, sur une terrasse plus basse que celle qui livre la sigillée; il peut donc s'agir d'un site de faible superficie, qui s'est déplacé. Là encore, le recours à des données extérieures à l'analyse ajoute à la singularité du cas: outre la question de la superficie, on soulignera que c'est aux abords de cet habitat qu'a été trouvée la tombe du mas de Jallon, du début du $\mathrm{I}^{\mathrm{rr}} \mathrm{s}$. av. J.-C. ${ }^{16}$.

Au terme de l'analyse des données dont on vient d'exposer les résultats, il nous paraît utile d'évoquer les conditions qui ont permis d'aboutir à une typologie satisfaisante pour l'archéologue et le statisticien, et de tirer les leçons du "colloque invisible" qui nous a mobilisés pendant plus d'une année.

Cette analyse est le fruit d'une collaboration entre chercheurs objectivement éloignés : mais la distance qui sépare la Franche-Comté du Languedoc n'a aucunement constitué une gêne, dans la mesure où le traitement a été conduit de manière parallèle, à Besançon et à Lunel, sur des micro-ordinateurs personnels largement accessibles (APPLE IIe), avec le concours de logiciels de mise en œuvre facile et

16 P. Garmy, A. Michilozzi, P. Py, Une nouvelle sépulture protohistorique à Beaucaire (Gard) : la tombe du mas de Jallon, Reıue Archéologique de Narbonnaise, XIV, 1981, p. 71-87. 
rapide, conçus pour être maniés par des chercheurs en Lettres et Sciences Humaines, et permettant de maitriser tout le processus d'analyse, de la saisie du fichier de données à l'édition des graphes de l'AFC ou des arbres de la classification. C'est ce qui explique notre volonté de présenter ici les produits mêmes de ces logiciels, afin d'illustrer le confort qu'ils garantissent au chercheur peu disposé à consacrer du temps à l'exécution manuelle de graphes.

L'analyse présentée ici est également le fruit d'une collaboration entre chercheurs de disciplines différentes: ce qui signifie qu'à chaque étape du traitement s'est instauré un réel débat entre l'archéologue attaché au contenu des descripteurs retenus, eux-mêmes objet de discussion entre archéologues, et le statisticien, néophyte en la matière et avant tout sensible à la structure et au comportement des données, en dehors même de leurs enjeux sémantiques; le troisième partenaire jouant le rôle d'interface et de logographe, défenseur à la fois des intérêts de l'archéologie et des exigences de l'analyse des données.

Il faut souligner à cet égard que l'essentiel du temps a été consacré à l'analyse commune des résultats de l'AFC et de la classification, ce qui est une autre manière de rendre hommage au travail de chercheurs comme ceux du Laboratoire Mathématique, Informatique et Statistique : quand ils conçoivent un logiciel, l'objectif à atteindre est de permettre au béotien d'acquérir le plus vite possible son indépendance par rapport à l'outil, pour qu'il puisse consacrer son énergie aux vrais problèmes.

Dégagés d'une quelconque dépendance technique, nous avons pu donc entamer le réel débat, celui de l'analyse des données. C'est ce type même de fonctionnement, où chacun était l'égal de l'autre face aux graphes et aux arbres produits par le microordinateur, qui a permis à l'analyse des données de jouer son rôle contestateur, en dérangeant les certitudes acquises et en heurtant les présupposés initiaux. Il eût été possible d'utiliser les résultats obtenus pour conforter la typologie initiale. Mais dès lors qu'on avait l'ambition d'intégrer toutes les leçons des graphes, et singulièrement la place qu'ils assignent aux descripteurs paysagers dans la différenciation des subdivisions de classes, il convenait d'accepter la singularité des cas qui contredisent la cohérence de tel ou tel groupe. Il importait surtout de chercher à comprendre ce qui génère la singularité, ce qui peut expliquer le comportement curieux de tel ou tel site. Il fallait accepter finalement de remettre en cause l'information initiale, d'interroger les conditions de production des données soumises à l'analyse et d'évaluer leur qualité intrinsèque. La synthèse suffit à illustrer le fonctionnement de cette démarche: l'analyse des données a contribué à mettre en évidence des sites inclassables, mais c'est l'archéologie qui peut seule donner la clé de l'impossibilité pour les sites concernés de s'intégrer complètement et de manière satisfaisante dans un des groupes définis au terme de l'analyse. Au total, l'analyse des données a constitué une étape importante dans la validation des descripteurs retenus; elle a prouvé l'intérêt d'inscrire les sites dans leur environnement paysager et de faire intervenir des descripteurs extra-archéologiques.

Ces résultats nous invitent à tester ces descripteurs ailleurs. Des paysages différents, des prospections plus systématiques, des échantillons de matériel plus variés devraient permettre de compléter et d'affiner les descripteurs. Mais le recours à une même démarche analytique assurera l'approfondissement des nécessaires comparaisons micro-régionales. Les opérations actuellement en cours autour de Donzère (Drôme) et dans les cantons de Lunel et de Mauguio (Hérault) nourrissent d'importants fichiers de données, dont l'analyse permettra de dégager les constantes typologiques ainsi que les différences régionales, qui ne s'esquissent aujourd'hui que de façon subjective.

$$
\begin{gathered}
\text { François Favory* } \\
\text { Jean-Luc Fiches** } \\
\text { Jean-Jacques GirardoT*** }
\end{gathered}
$$

* UA 338 "Analyse des Formations sociales de l'Antiquité»; GS 36 "Techniques nouvelles en Sciences de l'Homme" (Besançon).

** UA 284 "Centre Camille-Jullian" (Aix-en-Provence).

*** Laboratoire de Mathématique, Informatique et Statistique (MIS); GS 36 «Techniques nouvelles en Sciences de l'Homme», 30, rue Mégevand, 25030 Besançon Cedex (81.82.25.01).

N.B. - Les figures 1 et 13 sont l'œuvre de M. Borely, Centre Camille-Jullian. 


\section{APPENIDICE}

Découvertes et sites archéologiques du Beaucairols (fig. 13)

Commune.

\section{Beaucaire}

Beaucaire

Beaucaire

Beaucaire

Beaucaire

Beaucaire

Beaucaire

Beaucaire

Beaucaire

Beaucaire

Beaucaire

Beaucaire

Beaucaire

Beaucaire

Beaucaire

Beaucaire

Beaucaire

Beaucaire

Beaucaire

Beaucaire

Beaucaire

Beaucaire

Beaucaire

Bellegarde

Bellegarde

Bellegarde

Bellegarde

Bellegarde

Bellegarde

Comps

Jonquières-Saint-Vincent

Jonquières-Saint-Vincent

Jonquières-Saint-Vincent

Jonquières-Saint-Vinvent

Jonquières-Saint-Vincent

Jonquières-Saint-Vincent

Jonquières-Saint-Vincent

Jonquières-Saint-Vincent

Manduel

Manduel

Beaucaire

Beaucaire

Beaucaire
Site

Saint-Denis

Valescure

Saint-Pierre-de-Campublic

Tourelles

Pauvre-Ménage

Site $n^{\circ} 1$

Mas-.Veuf

Privat

Les Arves

Mas-de-Mailland

Mourillon

Mas-de-Bosc

Saint-Paul

Malatrache

Site n" 1

Site $n^{\prime \prime} 2$

Genestet

Ioton

Mas-de-Peyre

Mas-Peirette

Mas-de-Marquet

Saint-IIenri

Le Contrac

Mas-de-Roustan

Briquet

Broussan

Pièchegut

Mas-de-Bions

Carron-du-Contrac

Mas-Saint-Jean

Pont-de-Servant

Les Lônes

Fontaine-de-Tavie

Las Planes

Fontanille

Les Cinquains

La Tombe

Le Grand Vallat

La Tine

Mas-de-Perset

Mas-de-Carlot

Site n" 2

Site $n^{\circ} 2$

Site $n^{\circ} 3$
Lieu-dit

Canton d'Arles

Valescure

Mas-de-Bosc

Tour-à-Saint-Pierre

Les Tourelles

Mas-de-Forton Nord

La-Font-du-Rey

Mas-.Neuf

Mas-de-Martin

Mas-de-Forton Nord

Les Arves

Mas-de-Mailland

Saint-Roman Ouest

Mas-de-Bosc

Le Domptaïre

Malatrache

Mas-de-Moutet

Mas-de-Moutet

Genestet Ouest

Ioton

Mas-de-Peyre

Mas-de-Mailland

Mas-de-Marquet

Mas-de-Gleize

Le Contrac

Repentir

Briquet

Broussan

Pièchegut

Travers-de-Bions

Carron-du-Contrac

Pendant-de-Saint-Jean

Pont-de-Servant

Les Lònes

Fontaine-de-Tavie

Las Planes

Fontanille

Les Cinquains

La Tombe

Le Grand Vallat

La Tine

Mas-de-Perset

Caveau

Millas Sud

Valescure

Font-du-Rey

Font-du-Rey 